\title{
Direct Chargino-Neutralino Production at the LHC: Interpreting the Exclusion Limits in the Complex MSSM
}

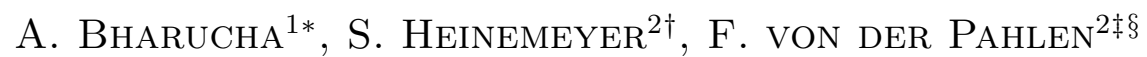 \\ ${ }^{1}$ II. Institut für Theoretische Physik, Universität Hamburg, Lupurer Chaussee 149, \\ D-22761 Hamburg, Germany \\ ${ }^{2}$ Instituto de Física de Cantabria (CSIC-UC), E-39005 Santander, Spain
}

\begin{abstract}
We re-assess the exclusion limits on the parameters describing the supersymmetric (SUSY) electroweak sector of the MSSM obtained from the search for direct charginoneutralino production at the LHC. We start from the published limits obtained for simplified models, where for the case of heavy sleptons the relevant branching ratio, $\operatorname{BR}\left(\tilde{\chi}_{2}^{0} \rightarrow \tilde{\chi}_{1}^{0} Z\right)$, is set to one. We show how the decay mode $\tilde{\chi}_{2}^{0} \rightarrow \tilde{\chi}_{1}^{0} h$, which cannot be neglected in any realistic model once kinematically allowed, substantially reduces the excluded parameter region. We analyze the dependence of the excluded regions on the phase of the gaugino soft SUSY-breaking mass parameter, $M_{1}$, on the mass of the light scalar tau, $m_{\tilde{\tau}_{1}}$, on $\tan \beta$ as well as on the squark and slepton mass scales. Large reductions in the ranges of parameters excluded can be observed in all scenarios. The branching ratios of charginos and neutralinos are evaluated using a full NLO calculation for the complex MSSM. The size of the effects of the NLO calculation on the exclusion bounds is investigated. We furthermore assess the potential reach of the experimental analyses after collecting $100 \mathrm{fb}^{-1}$ at the LHC running at $13 \mathrm{TeV}$.
\end{abstract}

*email: Aoife.Bharucha@desy.de

†email: Sven.Heinemeyer@cern.ch

† email: pahlen@ifca.unican.es

$\S$ MultiDark Fellow 


\section{Introduction}

The LHC is actively searching for physics beyond the Standard Model (BSM). Many of those searches rely on the predictions of specific models. A well motivated model is the Minimal Supersymmetric Standard Model (MSSM) [1], which provides a framework in which such predictions can be made. Provided $R$ parity is conserved [2], the final particle of any SUSY decay chain is the lightest supersymmetric particle (LSP), e.g. the lightest neutralino. It was shown that this particle is a natural candidate for Cold Dark Matter (CDM) 3]. The MSSM also contains a rich Higgs phenomenology, particularly relevant in light of the exciting recent discovery by ATLAS and CMS of a scalar resonance at $\sim 125 \mathrm{GeV}$ [4], as the requirement of an additional Higgs doublet results in a total of five physical Higgs bosons, the light and heavy $\mathcal{C P}$-even Higgs bosons, $h$ and $H$, the $\mathcal{C P}$-odd $A$ and the charged Higgs bosons, $H^{ \pm}$.

The search for supersymmetry (SUSY) at the LHC has not (yet) led to a positive result. In particular, bounds on the first and second generation squarks and the gluinos from ATLAS and CMS are very roughly at the TeV scale, depending on details of the assumed parameters, see e.g. [5]. On the other hand, bounds on the electroweak SUSY sector, where $\tilde{\chi}_{1,2}^{ \pm}$and $\tilde{\chi}_{1,2,3,4}^{0}$ denote the charginos and neutralinos (i.e. the charged (neutral) SUSY partners of the SM gauge and Higgs bosons) are substantially weaker. Here it should be noted that models based on Grand Unified Theories (GUTs) naturally predict a lighter electroweak spectrum (see Ref. [6] and references therein). Furthermore, the anomalous magnetic moment of the muon shows a more than $\sim 4 \sigma$, deviation from the SM prediction, see Ref. [7] and references therein. Agreement of this measurement with the MSSM requires charginos and neutralinos in the range of several hundreds of $\mathrm{GeV}$. This provides a strong motivation for the search of these electroweak particles, which could be in the kinematic reach of the LHC. One promising channel is the direct production of a chargino and neutralino, $p p \rightarrow \tilde{\chi}_{1}^{ \pm} \tilde{\chi}_{2}^{0}(+X)$. Although the cross sections are generically lower than for the direct production of colored particles, the searches at ATLAS and CMS have lead to several limits in the range of the order of several hundreds of $\mathrm{GeV}$, see e.g. Refs. [8, 9], which are independent of the mass scale of colored SUSY particles. The highest sensitivity comes from multi-lepton final states, including tau leptons, which offer the possibility to distinguish a signal from the large hadronic background. As an additional advantage the theoretical calculation is cleaner than that of the production of electroweak SUSY particles via cascade decays of colored particles.

Early on, studies had proposed the clean trilepton signal at the LHC, coming either from intermediate sleptons (particularly of the first and second generation) or gauge boson decays to which the experiments are more sensitive (see e.g. Ref. [10]). More recently there have been several studies investigating the LHC sensitivity to this decay mode, e.g. in the region where decays to trileptons via $W$ and $Z$ bosons dominate [11. Direct production of electroweak SUSY particles has also been investigated in more challenging scenarios where the lightest chargino and two neutralinos are higgsino-like, and thus nearly degenerate, such that their decay signals are lost in the SM background [12], but a same sign diboson signal from gaugino production could be detectable for wino masses up to $550 \mathrm{GeV}$ with $100 \mathrm{fb}^{-1}$ at LHC14 [13]. Other recent studies have focused on improving the reach of searches using e.g. a kinematic observable, the visible transverse energy for $W Z+E_{T}^{\text {miss }}$ final states [14]. Furthermore, there has been an increasing interest in the $W h+E_{T}^{\text {miss }}$ final state [15], including a $h \rightarrow b \bar{b}$ decay, for which the use of jet substructure was found to improve results [16, 17]. 
This improvement is particularly helpful in gauge-mediated scenarios, in which the GUT relation is broken $\left(M_{2} \nsim 2 M_{1}\right)$, and boosted Higgs bosons could be observed with $15 \mathrm{fb}^{-1}$ at LHC14 for values of $M_{2} \sim 200 \mathrm{GeV}-300 \mathrm{GeV}$ [16].

As discussed before, ATLAS [8, 18, 21] and CMS [9,22] are actively searching for the direct production of charginos and neutralinos, in particular for the process $p p \rightarrow \tilde{\chi}_{1}^{ \pm} \tilde{\chi}_{2}^{0}$ with the subsequent decays $\tilde{\chi}_{1}^{ \pm} \rightarrow \tilde{\chi}_{1}^{0} W^{ \pm}$and $\tilde{\chi}_{2}^{0} \rightarrow \tilde{\chi}_{1}^{0} Z$, resulting a three lepton signature. These searches are performed mostly in so-called "simplified models", where the branching ratios of the relevant SUSY particles are set to one, assuming that all other potential decay modes are kinematically forbidden. The results are (often) presented in the $m_{\tilde{\chi}_{2}^{0}}-m_{\tilde{\chi}_{1}^{0}}$ parameter plane.

To compare with these experimental results, precise predictions for the $W Z+E_{T}^{\text {miss }}$ final state are required, involving both calculations for the gaugino production cross section and the branching ratios of the subsequent chargino and neutralino decays. The production cross section in the $\mathcal{C} \mathcal{P}$-conserving real MSSM (rMSSM) was calculated at NLO and incorporated into the code Prospino 2.1 [23], as well as at NLL accuracy and investigated in the context of the LHC\&1 in Ref. [24]. Chargino and neutralino cross sections for the LHC8 in the complex MSSM have not been analyzed so far. Chargino and neutralino decays have been calculated at the one-loop level in the rMSSM [26 29] and in the complex MSSM [30 35], where our evaluations are based on the first full one-loop (NLO) calculation (of all non-hadronic decays) presented in Refs. [33,34]. A phenomenological analysis in the complex MSSM, where these state-of-the-art results are combined to make predictions for the LHC is still lacking. Turning to the neutralino decays, in Ref. 34] the NLO results for all possible neutralino decays were considered as a function of $\varphi_{M_{1}}$, under the assumption that colored particles are kinematically excluded. It was found that a change of the phase of $M_{1}=\left|M_{1}\right| e^{i \varphi_{M_{1}}}$ can significantly alter the dominant decay mode when the decay modes to neutralinos and Higgs bosons are allowed. The NLO corrections have been found to be sizeable, particularly for channels involving Higgs bosons.

In this paper we define and analyze a set of scenarios for the production and decay of charginos and neutralinos at the LHC8, where we take $m_{\tilde{\chi}_{2}^{0}}$ and $m_{\tilde{\chi}_{1}^{0}}$ as free parameters. The starting point is the scenario used by ATLAS to present their results for $21 \mathrm{fb}^{-1}$ [20], which so far constitutes the most sensitive test of direct electroweak SUSY production. We show the effect on the chargino and neutralino searches of the inclusion of the decay $\tilde{\chi}_{2}^{0} \rightarrow \tilde{\chi}_{1}^{0} h$ (with $M_{h} \approx 125 \mathrm{GeV}$ ). Direct and indirect effects from decays to the recently discovered Higgs boson [4] must not be neglected in any realistic analysis. Subsequently, we deviate from the ATLAS scenario in several ways, motivated by current limits on the MSSM parameter space. In particular, we vary the phase of the gaugino soft SUSY-breaking mass parameter, $\varphi_{M_{1}}$, which has a strong impact on the branching ratios of the $\tilde{\chi}_{2}^{0}$ and thus on the limits of the exclusion regions in the $m_{\tilde{\chi}_{2}^{0}}-m_{\tilde{\chi}_{1}^{0}}$ plane. We furthermore analyze the scenario with a light scalar tau in the so-called $\tilde{\tau}$-coannihilation region, where the $\tilde{\chi}_{1}^{0}$ provides a good $\mathrm{CDM}$ candidate. We vary other parameters, such as $\tan \beta$ (the ratio of the two vacuum expectation values of the two Higgs doublets, $\left.\tan \beta=v_{2} / v_{1}\right)$, the higgsino mass parameter $\mu$, and the masses that set the scale for the scalar leptons or the scalar quarks. By analyzing these variations we aim to provide a more realistic interpretation of current ATLAS and

${ }^{1}$ With LHC $x$ we denote the LHC running at $\sqrt{s}=x \mathrm{TeV}$. 
CMS limits on the electroweak SUSY particles. Finally we investigate which limits can be expected from the first $100 \mathrm{fb}^{-1}$ at the LHC13 (i.e. with $\sqrt{s}=13 \mathrm{TeV}$ ), which could be obtained in the years 2015-2017.

The paper is organized as follows: We begin with a short review of the relevant parameters and couplings as well as the calculations employed in our analysis in Sec. 2, Then in Sec. 3 we review in more detail the existing experimental analyses and define the various scenarios in which the analysis will be performed. Sec. 4 contains the numerical results, i.e. the re-interpretation of the existing mass limits in the benchmark scenarios, as well as our extrapolation to the LHC13. We conclude in Sec. 5.

\section{Details of the calculation}

In this section, after having introduced the necessary notation, we will illustrate the dependence of the couplings on the fundamental parameters via a simple expansion, and then go on to describe the details of the calculations employed in our (NLO) analysis in Sec. 4.

\section{$2.1 \quad$ Notation}

In the chargino case, two $2 \times 2$ matrices $\mathbf{U}$ and $\mathbf{V}$ are necessary for the diagonalization of the chargino mass matrix $\mathbf{X}$,

$$
\mathbf{M}_{\tilde{\chi}^{-}}=\mathbf{V}^{*} \mathbf{X}^{\top} \mathbf{U}^{\dagger}=\left(\begin{array}{cc}
m_{\tilde{\chi}_{1}^{ \pm}} & 0 \\
0 & m_{\tilde{\chi}_{2}^{ \pm}}
\end{array}\right) \quad \text { with } \quad \mathbf{X}=\left(\begin{array}{cc}
M_{2} & \sqrt{2} \sin \beta M_{W} \\
\sqrt{2} \cos \beta M_{W} & \mu
\end{array}\right)
$$

where $\mathbf{M}_{\tilde{\chi}^{-}}$is the diagonal mass matrix with the chargino masses $m_{\tilde{\chi}_{1}^{ \pm}}, m_{\tilde{\chi}_{2}^{ \pm}}$as entries, which are determined as the (real and positive) singular values of $\mathbf{X}$ and $M_{W}$ is the mass of the $W$ boson. The singular value decomposition of $\mathbf{X}$ also yields results for $\mathbf{U}$ and $\mathbf{V}$.

In the neutralino case, as the neutralino mass matrix $\mathbf{Y}$ is symmetric, one $4 \times 4$ matrix is sufficient for the diagonalization

$$
\mathbf{M}_{\tilde{\chi}^{0}}=\mathbf{N}^{*} \mathbf{Y} \mathbf{N}^{\dagger}=\operatorname{diag}\left(m_{\tilde{\chi}_{1}^{0}}, m_{\tilde{\chi}_{2}^{0}}, m_{\tilde{\chi}_{3}^{0}}, m_{\tilde{\chi}_{4}^{0}}\right)
$$

with

$$
\mathbf{Y}=\left(\begin{array}{cccc}
M_{1} & 0 & -M_{Z} s_{\mathrm{w}} \cos \beta & M_{Z} s_{\mathrm{w}} \sin \beta \\
0 & M_{2} & M_{Z} c_{\mathrm{w}} \cos \beta & -M_{Z} c_{\mathrm{w}} \sin \beta \\
-M_{Z} s_{\mathrm{w}} \cos \beta & M_{Z} c_{\mathrm{w}} \cos \beta & 0 & -\mu \\
M_{Z} s_{\mathrm{w}} \sin \beta & -M_{Z} c_{\mathrm{w}} \sin \beta & -\mu & 0
\end{array}\right)
$$

$M_{Z}$ is the mass of the $Z$ boson, $c_{\mathrm{w}}=M_{W} / M_{Z}$ and $s_{\mathrm{w}}=\sqrt{1-c_{\mathrm{w}}^{2}}$. The unitary $4 \times 4$ matrix $\mathbf{N}$ and the physical neutralino (tree-level) masses $m_{\tilde{\chi}_{k}^{0}}(k=1,2,3,4)$ result from a numerical Takagi factorization [36] of $\mathbf{Y}$.

When working in the complex MSSM it should be noted that the results for physical observables are affected only by certain combinations of the complex phases of the parameters. It is possible, for instance, to rotate the phase $\varphi_{M_{2}}$ away, which we adopt here. In this case the phase $\varphi_{\mu}$ is tightly constrained [37]. Consequently, we take $\mu$ to be a real parameter. 
Further note that in the case of the complex MSSM, the three neutral Higgs bosons $h, H$ and $A$ mix at the loop level [38 41], resulting in the (mass ordered) $h_{1}, h_{2}$ and $h_{3}$, which are not states of definite $\mathcal{C P}$-parity. In the following we denote the light Higgs with $h_{1}$, independent whether the parameters are chosen complex or real. The Higgs sector predictions have been derived with FeynHiggs 2.9.4 [42,45].

\section{$2.2 \varphi_{M_{1}}$ dependence of neutralino amplitudes}

In this section we investigate the $\varphi_{M_{1}}$ dependence of amplitudes for $\tilde{\chi}_{2}^{0}$ decays in the limit $\mu \gg\left|M_{1}\right|, M_{2} ; M_{H^{ \pm}} \gg M_{Z} ; \tan \beta \gg 1$, which will be relevant for most of the analyzed benchmark scenarios. The full $\tilde{\chi}_{i}^{0} \tilde{\chi}_{j}^{0} Z / h_{1}$ couplings take the form (with $e$ denoting the electric charge, $\alpha_{\mathrm{em}}=e^{2} /(4 \pi)$, and $\alpha$ is the angle that diagonalizes the $\mathcal{C P}$-even Higgs sector at tree-level)

$$
\begin{aligned}
C_{\tilde{\chi}_{i}^{0} \tilde{\chi}_{j}^{0} Z}^{L} & =-\frac{e}{2 c_{\mathrm{w}} s_{\mathrm{w}}}\left[N_{i 3} N_{j 3}{ }^{*}-N_{i 4} N_{j 4}{ }^{*}\right] \\
C_{\tilde{\chi}_{i}^{0} \tilde{\chi}_{j}^{0} h_{1}}^{L} & =-\frac{e}{2 c_{\mathrm{w}} s_{\mathrm{w}}}\left[\left(\sin \alpha N_{i 3}{ }^{*}+\cos \alpha N_{i 4}{ }^{*}\right)\left(s_{\mathrm{w}} N_{j 1}{ }^{*}-c_{\mathrm{w}} N_{j 2}{ }^{*}\right)+(i \leftrightarrow j)\right],
\end{aligned}
$$

showing the left-handed ( $\mathrm{LH})$ parts, with the right-handed $(\mathrm{RH})$ parts following from hermiticity of the Lagrangian, see e.g. Ref. [46],

$$
C_{\tilde{\chi}_{i}^{0} \tilde{\chi}_{j}^{0} Z}^{R}=-C_{\tilde{\chi}_{i}^{0} \tilde{\chi}_{j}^{0} Z}^{L *}, \quad C_{\tilde{\chi}_{i}^{0} \tilde{\chi}_{j}^{0} h_{1}}^{R}=C_{\tilde{\chi}_{i}^{0} \tilde{\chi}_{j}^{0} h_{1}}^{L *} .
$$

In the limit of interest to us, the two lightest neutralinos are almost purely bino and wino-like states, $\tilde{\chi}_{1}^{0} \sim \tilde{B}, \tilde{\chi}_{2}^{0} \sim \tilde{W}$. Here we neglect the mixing between the bino and wino components, which has a subleading effect in our approximation, such that $N_{12} \simeq N_{21} \simeq 0$, while $\left|N_{11}\right| \simeq\left|N_{22}\right| \simeq 1$. Note that in the Higgs decoupling limit [47] one has $(\beta-\alpha) \rightarrow \pi / 2$. In this limit we obtain for Eqs. (44) and (5)

$$
\begin{aligned}
& C_{\tilde{\chi}_{1}^{0} \tilde{\chi}_{2}^{0} Z}^{L} \approx \frac{e}{2} \frac{M_{Z}^{2}}{\mu^{2}} \exp \left(\frac{i \varphi_{M_{1}}}{2}\right), \\
& C_{\tilde{\chi}_{1}^{0} \tilde{\chi}_{2}^{0} h_{1}}^{L} \approx \frac{e}{2} \frac{M_{Z}}{\mu}\left(\frac{M_{1}+M_{2}}{\mu}+\frac{4}{\tan \beta}\right) \exp \left(\frac{-i \varphi_{M_{1}}}{2}\right),
\end{aligned}
$$

where the neglected terms are of higher order in $M_{Z} / \mu, M_{1 / 2} / \mu$ and $1 / \tan \beta$. Eqs. (17) and (8) also show that the absolute value of the Higgs coupling is largest (smallest) for positive (negative) $M_{1}$. The partial decay widths, however, also depend on the relative intrinsic $\mathcal{C P}$ factor $\eta_{12}$ of the neutralinos and that of the Higgs boson. (the $Z$-boson is $\mathcal{C P}$-even). This effect leads to a larger (much larger near the threshold) dependence on the $\mathcal{C} \mathcal{P}$-phases than the one resulting from the change in the absolute value of the couplings, provided $m_{\tilde{\chi}_{1}^{0}} \neq 0$, as we illustrate below.

Using the relation (6) between the LH and RH couplings we express the tree-level partial decay widths as 


$$
\begin{aligned}
\Gamma^{\text {tree }}\left(\tilde{\chi}_{2}^{0} \rightarrow \tilde{\chi}_{1}^{0} Z\right)= & \frac{\beta^{*}\left(\tilde{\chi}_{1}^{0}, \tilde{\chi}_{2}^{0}, Z\right)}{16 \pi m_{\tilde{\chi}_{2}^{0}}^{0}}\left|C_{\tilde{\chi}_{1}^{0} \tilde{\chi}_{2}^{0} Z}^{L}\right|^{2} \\
& \times\left(m_{\tilde{\chi}_{2}^{0}}^{2}+m_{\tilde{\chi}_{1}^{0}}^{2}-2 M_{Z}^{2}+\frac{\left(m_{\tilde{\chi}_{2}^{0}}^{2}-m_{\tilde{\chi}_{1}^{0}}^{2}\right)^{2}}{M_{Z}^{2}}+6 \cos \left(\varphi_{\tilde{\chi}_{1}^{0} \tilde{\chi}_{2}^{0} Z}\right) m_{\tilde{\chi}_{2}^{0}} m_{\tilde{\chi}_{1}^{0}}\right) \\
\Gamma^{\operatorname{tree}}\left(\tilde{\chi}_{2}^{0} \rightarrow \tilde{\chi}_{1}^{0} h_{1}\right)= & \frac{\beta^{*}\left(\tilde{\chi}_{1}^{0}, \tilde{\chi}_{2}^{0}, h_{1}\right)}{16 \pi m_{\tilde{\chi}_{2}^{0}}}\left|C_{\tilde{\chi}_{1}^{0} \tilde{\chi}_{2}^{0} h_{1}}^{L}\right|^{2} \\
& \times\left(m_{\tilde{\chi}_{2}^{0}}^{2}+m_{\tilde{\chi}_{1}^{0}}^{2}-m_{h_{1}}^{2}+2 \cos \left(\varphi_{\tilde{\chi}_{1}^{0} \tilde{\chi}_{2}^{0} h_{1}}\right) m_{\tilde{\chi}_{2}^{0}} m_{\tilde{\chi}_{1}^{0}}\right)
\end{aligned}
$$

with

$$
\cos \left(\varphi_{\tilde{\chi}_{1}^{0} \tilde{\chi}_{2}^{0} Z}\right)=\frac{2 \operatorname{Re}\left\{C_{\tilde{\chi}_{1}^{0} \tilde{\chi}_{2}^{0} Z}^{L *} C_{\tilde{\chi}_{1}^{0} \tilde{\chi}_{2}^{0} Z}^{R}\right\}}{\left|C_{\tilde{\chi}_{1}^{0} \tilde{\chi}_{2}^{0} Z}^{L}\right|^{2}+\left|C_{\tilde{\chi}_{1}^{0} \tilde{\chi}_{2}^{0} Z}^{R}\right|^{2}}, \quad \cos \left(\varphi_{\tilde{\chi}_{1}^{0} \tilde{\chi}_{2}^{0} h_{1}}\right)=\frac{2 \operatorname{Re}\left\{C_{\tilde{\chi}_{1}^{0} \chi_{2}^{0} h_{1}}^{L *} C_{\tilde{\chi}_{1}^{0} \tilde{\chi}_{2}^{0} h_{1}}^{R}\right\}}{\left|C_{\tilde{\chi}_{1}^{0} \tilde{\chi}_{2}^{0} h_{1}}^{L}\right|^{2}+\left|C_{\tilde{\chi}_{1}^{0} \tilde{\chi}_{2}^{0} h_{1}}^{R}\right|^{2}}
$$

and $\beta^{*}(a, b, c)=\lambda^{1 / 2}\left(m_{a}^{2}, m_{b}^{2}, m_{c}^{2}\right) / m_{a}^{2}$, where $\lambda(x, y, z)=(x-y-z)^{2}-4 y z$. The coefficients defined by Eq. (11) are related to the relative $\mathcal{C P}$ phase factor of the particles involved. In this section we will further assume, for the sake of simplicity, that $h_{1}$ is $\mathcal{C P}$-even. However, the generalization to the general case is straightforward. When $M_{1}>0$ the relative $\mathcal{C P}$ parity of the neutralinos and the Higgs boson or $Z$ boson is positive, allowing this process in s-wave. When $M_{1}<0$ the situation is the opposite, with negative relative $\mathcal{C P}$ parity. In this case only odd-values of the total angular momentum allowed, leading to p-wave suppressed processes near the corresponding decay thresholds.

In the limit of interest the partial decay widths, Eqs. (9) and (10), are given by

$$
\begin{aligned}
\Gamma^{\text {tree }}\left(\tilde{\chi}_{2}^{0} \rightarrow \tilde{\chi}_{1}^{0} Z\right) \approx & \frac{e^{2} \beta^{*}\left(\tilde{\chi}_{1}^{0}, \tilde{\chi}_{2}^{0}, Z\right)}{64 \pi m_{\tilde{\chi}_{2}^{0}}} \frac{M_{Z}^{4}}{\mu^{4}} \\
& \times\left(m_{\tilde{\chi}_{2}^{0}}^{2}+m_{\tilde{\chi}_{1}^{0}}^{2}-2 M_{Z}^{2}+\frac{\left(m_{\tilde{\chi}_{2}^{0}}^{2}-m_{\tilde{\chi}_{1}^{0}}^{2}\right)^{2}}{M_{Z}^{2}}+6 \cos \left(\varphi_{M_{1}}\right) m_{\tilde{\chi}_{2}^{0}} m_{\tilde{\chi}_{1}^{0}}\right), \\
\Gamma^{\text {tree }}\left(\tilde{\chi}_{2}^{0} \rightarrow \tilde{\chi}_{1}^{0} h_{1}\right) \approx & \frac{e^{2} \beta^{*}\left(\tilde{\chi}_{1}^{0}, \tilde{\chi}_{2}^{0}, h_{1}\right)}{64 \pi m_{\tilde{\chi}_{2}^{0}}} \frac{M_{Z}^{2}}{\mu^{2}}\left|\frac{M_{1}+M_{2}}{\mu}+\frac{4}{\tan \beta}\right|^{2} \\
& \times\left(m_{\tilde{\chi}_{2}^{0}}^{2}+m_{\tilde{\chi}_{1}^{0}}^{2}-m_{h_{1}}^{2}+2 \cos \left(\varphi_{M_{1}}\right) m_{\tilde{\chi}_{2}^{0}} m_{\tilde{\chi}_{1}^{0}}\right)
\end{aligned}
$$

These expressions show explicitly the large $\varphi_{M_{1}}$ dependence of the tree-level partial decay widths to the Higgs boson, both from the couplings as well as from the relative $\mathcal{C P}$ of initial and final states.

\subsection{Calculation}

Here we briefly review the calculations used for the direct production cross section of $\tilde{\chi}_{2}^{0} \tilde{\chi}_{1}^{ \pm}$, and for the branching ratios for the subsequent decay of the neutralino into a $Z$ boson and of the chargino into a $W$ boson and the LSP. The main production channels for $\tilde{\chi}_{1}^{ \pm} \tilde{\chi}_{2}^{0}$ at the LHC, as well as their two-body decays to gauge and Higgs bosons and the neutralino decay to a tau-stau pair are shown in Figs. 1 and 2, respectively. 

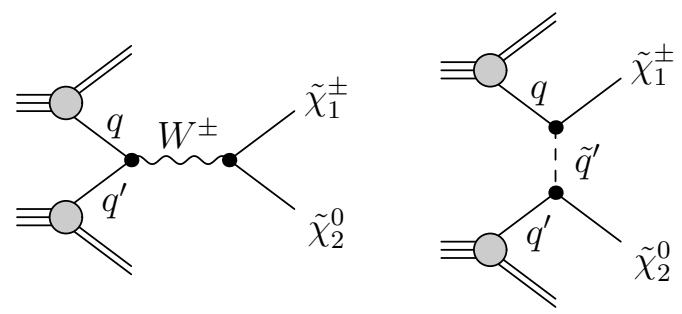

Figure 1: Main production channels for $\tilde{\chi}_{1}^{ \pm} \tilde{\chi}_{2}^{0}$ at the LHC. Here $q$ and $q^{\prime}\left(\tilde{q}^{\prime}\right)$ denote quarks (squarks) of the first generation.
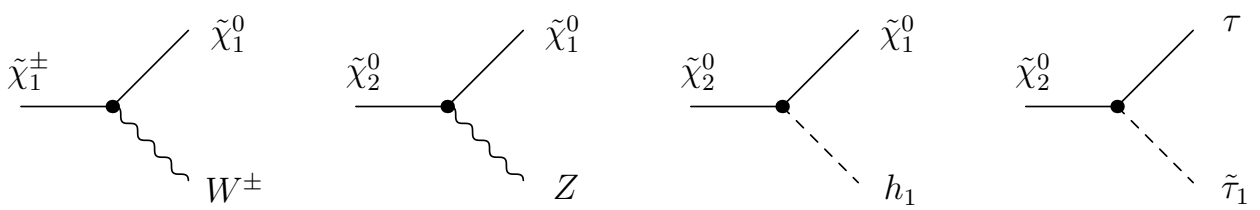

Figure 2: Two-body decay channels of $\tilde{\chi}_{1}^{ \pm}$and $\tilde{\chi}_{2}^{0}$ to gauge and Higgs bosons, as well as the neutralino decay to a tau-stau pair. It should be noted that these channels are kinematically allowed only in parts of the MSSM parameter space.

The production of neutralinos and charginos at the LHC is calculated using the program Prospino 2.1 [23]. The effect of complex parameters on these cross sections can only enter via chargino or neutralino mixing effects. We have evaluated these cross sections at the parton level to estimate its effect, which turns out to be negligible in our analysis2. Consequently, the Prospino results can be taken over also for the complex MSSM results. Small differences for the calculation of $p p \rightarrow \tilde{\chi}_{1}^{+} \tilde{\chi}_{2}^{0}$ and of $p p \rightarrow \tilde{\chi}_{1}^{-} \tilde{\chi}_{2}^{0}$ are neglected. The NLL corrections to the gaugino production cross section calculated in Ref. [24,25] are not included, and we estimate their effects to be at the per-cent level.

The production is dominated by wino pair production, where the largest contribution is from the $s$-channel gauge boson diagrams. If one assumes that $M_{1}<M_{2}$, as is the case when the GUT relation for the gaugino mass parameters holds, then the neutralino with the largest wino component is either the second lightest neutralino (for $M_{2}<\mu$ ) or the heaviest one. Therefore, $\tilde{\chi}_{2}^{0} \tilde{\chi}_{1}^{\mp}$ and $\tilde{\chi}_{1}^{+} \tilde{\chi}_{1}^{-}$will have the largest production cross sections. Note that although the $t$ and $u$-channel contribution to pair production are suppressed due to squark propagators if one assumes the first generation squarks to be heavy, the destructive interference of the $t$-channel with left-handed squark exchange and the $s$-channel gauge boson channel can be significant, as will be discussed in Sec. 4 .

In Refs. [33, 34] we have calculated the full one-loop (NLO) corrections to the branching ratios for all non-hadronic chargino and neutralino decays for arbitrary parameters in the complex MSSM. The calculation is based on FeynArts/Formcalc [48, 49], and the corresponding model file conventions [46] are used throughout. In particular, the results were analyzed and found to be reliable as a function of $\varphi_{M_{1}}$. We will employ this NLO calculation for our investigations. The benchmark scenarios defined in the following section are such that the decays $\tilde{\chi}_{1}^{ \pm} \rightarrow \tilde{\chi}_{1}^{0} W^{ \pm}$as well as $\tilde{\chi}_{2}^{0} \rightarrow \tilde{\chi}_{1}^{0} Z, \tilde{\chi}_{2}^{0} \rightarrow \tilde{\chi}_{1}^{0} h_{1}, \tilde{\chi}_{2}^{0} \rightarrow \tilde{\tau}_{1}^{ \pm} \tau^{\mp}$ are the only relevant ones. As analyzed in the previous subsection the decays of a wino-like $\tilde{\chi}_{2}^{0}$ to $\tilde{\chi}_{1}^{0} h_{i}$

\footnotetext{
${ }^{2}$ The same holds for the production $p p \rightarrow \tilde{\chi}_{1}^{+} \tilde{\chi}_{1}^{-}$.
} 
are most sensitive to $\varphi_{M_{1}}$ due to the relative $\mathcal{C P}$ between the bino-like $\tilde{\chi}_{1}^{0}$ and the wino-like $\tilde{\chi}_{2}^{0}$, which is controlled by $\varphi_{M_{1}}$. This, however, can be modified when loop corrections are taken into account as discussed in Sec. 4.6. Furthermore, the NLO corrections are largest for decays to Higgs bosons [34] and thus have to be taken into account in a precision analysis. The production cross sections and decay branching ratios have been evaluated numerically using the OpenStack infrastructure as described in Ref. [50].

\section{Benchmark scenarios and experimental motivation}

\subsection{Overview of current experimental results}

In Refs. [8, 9, 18 22, ATLAS and CMS have studied the sensitivity to electroweak gaugino pair production, particularly to the production of the second lightest neutralino and lightest chargino via multi-lepton signatures. Here the chargino and neutralino decay either via sleptons or via gauge bosons, depending on the slepton masses, which are parameterized via $x$ (where $\left.m_{\tilde{l}}=m_{\tilde{\chi}_{1}^{0}}+x\left(m_{\tilde{\chi}_{2}^{0}}-m_{\tilde{\chi}_{1}^{0}}\right)\right)$. Exclusion limits are then obtained within specific models, primarily simplified models which set all relevant branching ratios to one, assuming that all other channels are kinematically forbidden. The ATLAS results at $7 \mathrm{TeV}$ are presented in Ref. [8] for $4.7 \mathrm{fb}^{-1}$, and the updated results including $8 \mathrm{TeV}$ data are given in Refs. [18] for $13 \mathrm{fb}^{-1}$ and [19 21] for up to $21 \mathrm{fb}^{-1}$. An update of CMS including the $8 \mathrm{TeV}$ data was published in Ref. [22] for $9.2 \mathrm{fb}^{-1}$, where opposite sign (OS) dileptons inconsistent with a $Z$ boson are also studied. The $7 \mathrm{TeV}$ results were published in Ref. [9] for $4.98 \mathrm{fb}^{-1}$.

In addition to the 3 lepton events (electrons, muons and hadronically reconstructed taus) analyzed by ATLAS, CMS also considers the case when one of the leptons is unidentified, selecting events with same the sign (SS) lepton pairs $e \tau, \mu \tau$ and $\tau \tau$. Further, OS lepton pairs and 2 jets for on-shell $W Z$ and $Z Z$ events where one $Z$ decays to $e^{+} e^{-}$or $\mu^{+} \mu^{-}$and the other gauge boson decays hadronically are considered. Simplified models are used to obtain exclusion limits, tuned to search for decays via sleptons or gauge boson, mainly for $x=0.5$ but also for $x=0.05$ and 0.95 . Here models with different couplings to $\tau$ leptons are considered, i.e. the sleptons may be left-handed or right-handed or a mixture, such that the final state leptons are predominantly light, flavor independent, or mostly taus.

ATLAS, on the other hand, presents its results for this channel by combining 3-lepton (electrons or muons) searches in various signal regions, the primary criterion being whether the invariant mass same-flavor-opposite-sign (SFOS) lepton pair lies around the $Z$ boson mass or not, thus defining $Z$-enriched and $Z$-depleted regions respectively. By making requirements on the reconstructed mass of the SFOS lepton pair $\left(m_{\text {SFOS }}\right)$ and on the transverse momentum $\left(p_{T}\right)$ of the third lepton, the depleted region is further subdivided into regions targeting either small mass splittings between the neutralinos (here the $Z$ is off-shell and this region is discussed later), mass splittings close to the $Z$-boson mass, or decays via sleptons by requiring high transverse momentum of the third-leading lepton. In the simplified models, a number of assumptions are made, first and foremost that the neutralino and chargino are wino-like and the lightest neutralino bino-like. As for the sleptons, either $x=0.5$ in which case the branching ratio to all sleptons is assumed to be $1 / 6$, or $x$ is very large (where the precise value is not quoted) such that the decay to sleptons may be ignored, and the branching ratio to gauge bosons is assumed to be 1 . 


\subsection{Definition of benchmark scenarios}

Since so far only ATLAS reported an analysis using the full 2012 data set with numerical values for the excluded cross sections [20, we will use their results for our baseline analysis. In order to interpret the ATLAS exclusions in terms of the complex MSSM, we calculate the cross section in benchmark scenarios similar to those used by ATLAS, including NLO corrections as described in Sec. 2.3. We re-analyze the ATLAS 95\% CL exclusion bounds in the simplified analyses in the $m_{\tilde{\chi}_{2}^{0}}-m_{\tilde{\chi}_{1}^{0}}$ plane, taking $M_{1}$ and $M_{2}$ as free parameters with central values:

$$
M_{1}=100 \mathrm{GeV} \text { and } M_{2}=250 \mathrm{GeV} .
$$

The other parameters are chosen as in the ATLAS analysis presented in Ref. [20]3,

$$
\mu=1 \mathrm{TeV}, \tan \beta=6, M_{\tilde{q}_{1,2}}=M_{\tilde{q}_{3}}=M_{\tilde{\ell}}=2 \mathrm{TeV}, A_{t}=2.8 \mathrm{TeV} .
$$

$M_{\tilde{q}_{1,2}}$ denotes the diagonal soft SUSY-breaking parameter in the scalar quark mass matrices of the first and second generation, similarly $M_{\tilde{q}_{3}}$ for the third generation and $M_{\tilde{\ell}}$ for all three generations of scalar leptons. If all three mass scales are identical we also use the abbreviation $M_{\mathrm{SUSY}}:=M_{\tilde{q}_{1,2}}=M_{\tilde{q}_{3}}=M_{\tilde{\ell}}$. We will clearly indicate where we deviate from the "unification" for scalar leptons. $A_{t}$ is the trilinear coupling between stop quarks and Higgs bosons, which is chosen to give the desired value of $M_{h_{1}}$. The other trilinear couplings, set to zero in Ref. [18,20], we set to $A_{t}$ for squarks and to zero for sleptons. Setting also the $A_{q \neq t}$ to zero would have a minor impact on our analysis. The effect the large sfermion mass scale is a small destructive interference of the $s$-channel amplitude with the $t$-channel squark exchange. The large higgsino mass parameter $\mu$ results in a gauginolike pair of produced neutralino and chargino. The lightest Higgs boson mass (as calculated with FeynHiggs 2.9.4 [42 [45]) is evaluated to be $\sim 125.5 \mathrm{GeV}$, defining the value of $A_{t}$ in Eq. (15). In order to scan the $m_{\tilde{\chi}_{2}^{0}}-m_{\tilde{\chi}_{1}^{0}}$ plane we use the ranges

$$
\left|M_{1}\right|=0 \ldots 200 \mathrm{GeV}, \quad M_{2}=100 \ldots 400 \mathrm{GeV} \text { with }\left|M_{1}\right| \leq M_{2} .
$$

The main aim of this paper, as discussed above, is the interpretation of the ATLAS exclusion limits in several "physics motivated" benchmark scenarios. Taking the parameters in Eq. (15) as our baseline scenario, we deviate from it in the following directions.

1. We take $\varphi_{M_{1}}$, the phase of $M_{1}$, to be a free parameter. Note that for the considered central benchmark scenario, as $\tan \beta$ is low and $M_{\text {SUSY }}$ is high, the full range is allowed by current electric dipole moment (EDM) constraints [51-53], as verified explicitly via both CPsuperH 2.3 [54 56] and FeynHiggs 2.9.4 [42 45].

2. The variation of $\tan \beta$ can have a strong impact on the couplings between the neutralinos and the Higgs boson, see Eq. (8) . We therefore analyze the effect of variation of $\tan \beta$ in the range $\tan \beta=6 \ldots 20$.

${ }^{3}$ Not all parameters are clearly defined in Ref. [20. We select and choose our parameters to be as close to the original analysis as possible. 
3. Although in general the sleptons are assigned a common mass $M_{\mathrm{SUSY}}$, in order to consider the possibility that neutralino decays to sleptons could compete with the decays to $Z$ and Higgs bosons, we consider $M_{\tilde{\tau}_{R}}=\left|M_{1}\right|$, where $M_{\tilde{\tau}_{R}}$ denotes the "righthanded" soft SUSY-breaking parameter in the scalar tau mass matrix, see Eq. (87) in Ref. 34. This scenario is motivated by the measured relic density of dark matter. For this choice of parameters one finds $m_{\tilde{\chi}_{1}^{0}} \lesssim m_{\tilde{\tau}_{1}}$, i.e. the stau co-annihilation region. We have confirmed (using micrOMEGAs3.1 [57]) that in our scenario the relic density is in agreement 4 with the latest measurements presented by Planck [58] earlier this year, $\Omega^{\mathrm{DM}} h^{2}=0.1199 \pm 0.0027$.

4. As shown in Eqs. (7), (8) the lighter neutralino-Higgs couplings depend strongly on $\mu$, and consequently also the Born amplitudes. We investigate two scenarios: (i) $M_{2}<\mu$ with $\mu=2000 \mathrm{GeV}$. This scenario shows similar characteristics as the ATLAS baseline scenario, but decouples the $\mu$ parameter further, as will be discussed briefly in Sec. 4.6. (ii) $M_{2}>\mu$ with $\mu=100 \ldots 400 \mathrm{GeV}$. In this scenario the lighter neutralinos and chargino possess a substantial higgsino component.

5. Although $M_{\text {SUSY }}$ has a negligible impact on the decays of the electroweak SUSY particles to gauge bosons, it plays an important role in the production, as the $t$-channel squark exchange and the $s$-channel gauge boson exchange amplitudes interfere destructively. We consider the range from $1.2 \mathrm{TeV}$ to $3 \mathrm{TeV}$.

The various scenarios are summarized in Tab. 1

Besides interpreting the current results in the scenarios summarized in Tab. 1 we also evaluate possible future limits (assuming the absence of a signal). We analyze the following two (future) scenarios:

(i) A combination of ATLAS and CMS data, which for simplicity we take as resulting in a doubling of the luminosity, i.e. assuming $42 \mathrm{fb}^{-1}$ analyzed by ATLAS. The change in the experimental limit on the production cross section (times branching ratio) is evaluated by assuming a purely statistical effect, thus dividing the current limit by $\sqrt{2}$.

(ii) The first run at $\sqrt{s}=13 \mathrm{TeV}$ that could take place in 2015-2017. We assume that ATLAS collects $100 \mathrm{fb}^{-1}$. The new limit is evaluated from the existing limits by a simple rescaling of signal and background cross sections. More details are given in Sec. 4.7.

\section{Interpretation of ATLAS exclusion limits}

We re-analyze the ATLAS results of Ref. [20] in the scenarios defined in Tab. 1. In all scenarios the decay $\tilde{\chi}_{2}^{0} \rightarrow \tilde{\chi}_{1}^{0} h_{1}$ is taken into account. Production cross sections and branching ratios are evaluated as described in Sec. 2.3. Note that in the simplified model analyses,

\footnotetext{
${ }^{4}$ Small changes in the parameters which can have a drastic impact on the predicted CDM density, but only a small impact on the chargino/neutralino phenomenology are not relevant.
} 


\begin{tabular}{|c||c|c|c|c|c|}
\hline Scenario & $\varphi_{M_{1}}$ & $\mu$ & $\tan \beta$ & $M_{\text {SUSY }}$ & $M_{\tilde{\tau}_{R}}$ \\
\hline \hline$S_{\text {ATLAS }}$ & 0 & 1000 & 6 & 2000 & $M_{\text {SUSY }}$ \\
\hline \hline$S_{\text {ATLAS }}^{\varphi_{M_{1}}}$ & $0 \ldots \pi$ & 1000 & 6 & 2000 & $M_{\text {SUSY }}$ \\
\hline$S_{\text {ATLAS }}^{\tan \beta}$ & 0 & 1000 & $6 \ldots 20$ & 2000 & $M_{\text {SUSY }}$ \\
\hline$S_{\text {ATLAS }}^{\mu}$ & 0 & 2000 & 6 & 2000 & $M_{\text {SUSY }}$ \\
\hline$S_{\text {ATLAS }}^{\text {SUS }}$ & 0 & 1000 & 6 & 1200,3000 & $M_{\text {SUSY }}$ \\
\hline \hline$S^{\text {DM }}$ & $0 \ldots \pi$ & 1000 & 6,20 & 2000 & $\left|M_{1}\right|$ \\
\hline$S_{\text {low }-\mu}$ & 0 & $100 \ldots 400$ & 6 & 2000 & $M_{\text {SUSY }}$ \\
\hline
\end{tabular}

Table 1: Parameters for benchmark scenarios (masses in $\mathrm{GeV}$ ). We furthermore have for all scenarios: $\left|M_{1}\right|=0 \ldots 200 \mathrm{GeV}, M_{2}=100 \ldots 400 \mathrm{GeV}$ (with $\left|M_{1}\right| \leq M_{2}$ ), $M_{3}=1500 \mathrm{GeV}$ (gluino mass parameter), except for the intermediate higgsinos scenario $S_{\mathrm{low}-\mu}$, where we set $M_{2}=500 \mathrm{GeV}$. The first (baseline) scenario corresponds to the ATLAS analysis in Ref. [20]. Our "central benchmark scenario" refers to the case $M_{1}=100 \mathrm{GeV}$ and $M_{2}=250 \mathrm{GeV}$. The value of $A_{t}$ is adjusted to ensure $M_{h_{1}} \approx 125.5 \mathrm{GeV}$.

$m_{\tilde{\chi}_{2}^{0}}=m_{\tilde{\chi}_{1}^{ \pm}}$, which is not the case in the MSSM. However, for lighter gauginos, corresponding to $|\mu|>\left|M_{1}\right|, M_{2}$, this relation holds to a good approximation. In our analysis, we chose to take the convention that our $m_{\tilde{\chi}_{2}^{0}}$ corresponds to the ATLAS $m_{\tilde{\chi}_{2}^{0}}$, and our $m_{\tilde{\chi}_{1}^{ \pm}}$is calculated accordingly. Note that in almost all of the parameter space explored the difference $m_{\tilde{\chi}_{2}^{0}}-m_{\tilde{\chi}_{1}^{ \pm}}$ will be less than $1 \mathrm{GeV}$, where larger values are indicated explicitly.

\subsection{Effect of $\tilde{\chi}_{2}^{0}$ decays to Higgs bosons and sign of $M_{1}$}

We begin by re-interpreting the ATLAS simplified model exclusion bounds, taking full NLO branching ratios into account. Including $\tilde{\chi}_{2}^{0} \rightarrow \tilde{\chi}_{1}^{0} h_{1}$ has a considerable impact on the $\operatorname{BR}\left(\tilde{\chi}_{2}^{0} \rightarrow \tilde{\chi}_{1}^{0} Z\right)$, thus weakens the existing conclusion limits. Keeping $M_{1}$ real, we analyze the excluded parameters allowing two possibilities for $\varphi_{M_{1}}$, namely 0 and $\pi$. (The case of a complex $M_{1}$ will be addressed in Sec. 4.3.)

In Fig. 3 we show as a black line the production cross section for $\tilde{\chi}_{1}^{ \pm} \tilde{\chi}_{2}^{0}$ pair-production at the LHC8 as a function of $m_{\tilde{\chi}_{2}^{0}}$ and for $m_{\tilde{\chi}_{1}^{0}}=0,50,100,150 \mathrm{GeV}$ in the upper right, upper left, lower right, lower left plot, respectively 5 The parameters are chosen according to $S_{\text {ATLAS }}$, except for $M_{1}$ and $M_{2}$ which are varied. In order to avoid $m_{\tilde{\chi}_{1}^{0}} \approx m_{\tilde{\chi}_{2}^{0}}$ the lines in the lower plots start at $m_{\tilde{\chi}_{2}^{0}}=125 \mathrm{GeV}$ (left) and $m_{\tilde{\chi}_{2}^{0}}=175 \mathrm{GeV}$ (right). The production cross section times the $\operatorname{BR}\left(\tilde{\chi}_{2}^{0} \rightarrow Z \tilde{\chi}_{1}^{0}\right)$ is also shown for $\varphi_{M_{1}}=0$ (green) and $\varphi_{M_{1}}=\pi$ (blue), except for $m_{\tilde{\chi}_{1}^{0}} \approx M_{1}=0.2 \mathrm{GeV}$ (upper left plot), where the sign is irrelevant. The chargino decays with $100 \%$ as $\tilde{\chi}_{1}^{ \pm} \rightarrow \tilde{\chi}_{1}^{0} W^{ \pm}$. The dashed green and blue lines correspond to the tree-level evaluation, whereas the solid lines are obtained by our full one-loop evaluation.

\footnotetext{
${ }^{5}$ It should be noted that a massless neutralino is not excluded by experimental searches [59].
} 

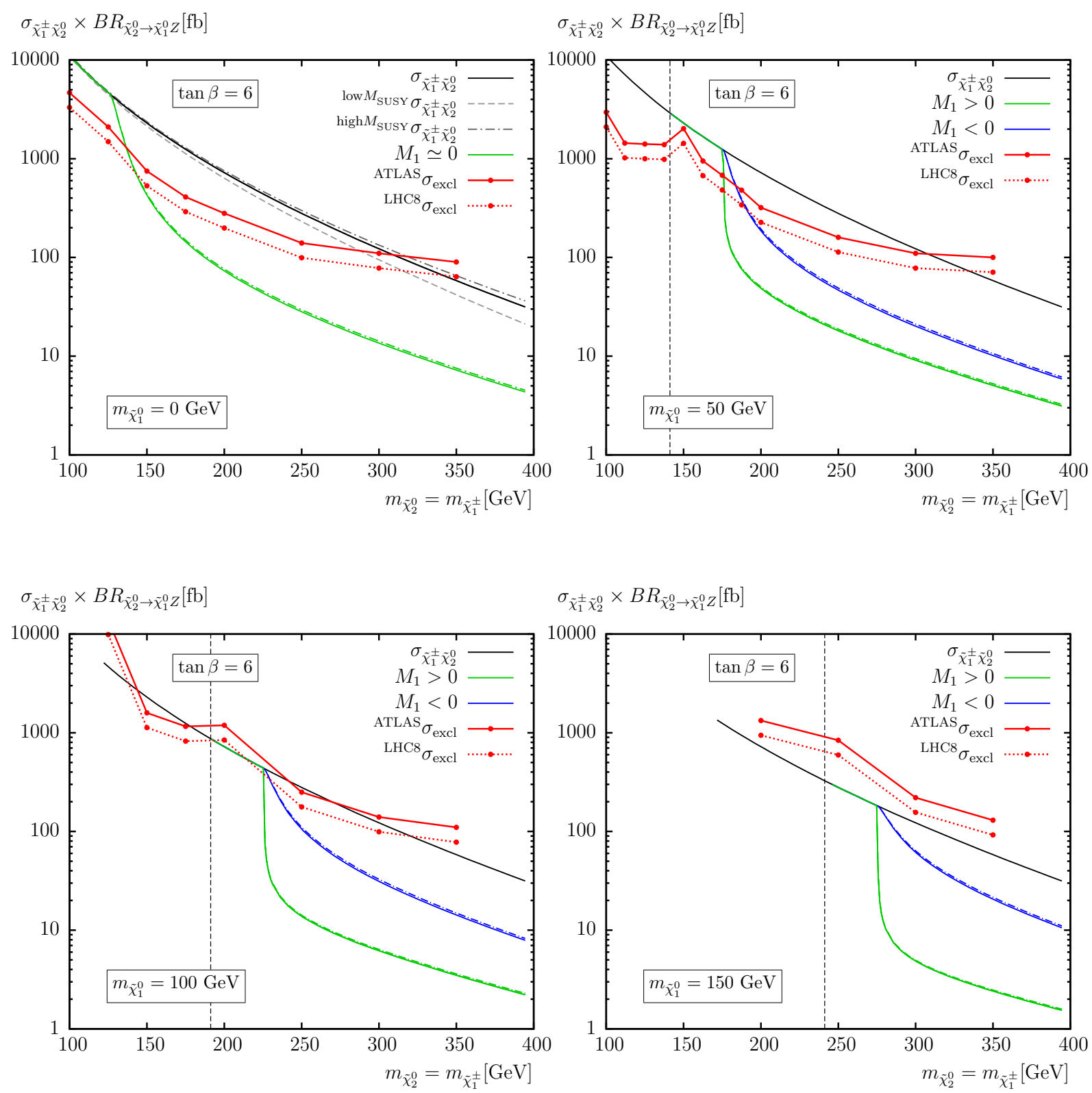

Figure 3: $\quad \tilde{\chi}_{1}^{ \pm}, \tilde{\chi}_{2}^{0}$ production cross section at the LHC8 (black) times the $\operatorname{BR}\left(\tilde{\chi}_{2}^{0} \rightarrow Z \tilde{\chi}_{1}^{0}\right)$ at tree (solid) and one-loop level (dashed-dotted) for $\varphi_{M_{1}}=0$ (green) and $\varphi_{M_{1}}=\pi$ (blue). $M_{1}$ is chosen such that $m_{\tilde{\chi}_{1}^{0}}=0,50,100,150 \mathrm{GeV}$, in the top left and right, bottom left and right plot, respectively. The 95\% CL exclusion cross sections from ATLAS taken from Fig. 8 and 9 of [20] (red, solid) are projected for the combined full data-set of ATLAS and CMS (red, dashed). Also shown in the upper left figure is the production cross section with $M_{\mathrm{SUSY}}=1.2 \mathrm{TeV}$ (dashed, light gray) and $M_{\mathrm{SUSY}}=3 \mathrm{TeV}$ (dot-dashed, dark gray). The vertical dashed lines show the position of the $\tilde{\chi}_{2}^{0} \rightarrow Z \tilde{\chi}_{1}^{0}$ kinematical threshold. 
The difference in Fig. 3, however, is barely visible. Below the kinematical threshold for $\tilde{\chi}_{2}^{0} \rightarrow \tilde{\chi}_{1}^{0} h_{1}$ the green/blue lines are on top of the black line, i.e. $\operatorname{BR}\left(\tilde{\chi}_{2}^{0} \rightarrow \tilde{\chi}_{1}^{0} Z\right)=1$. The green/blue lines stop at the dashed vertical line, indicating the kinematical threshold for $\tilde{\chi}_{2}^{0} \rightarrow \tilde{\chi}_{1}^{0} Z$. The $95 \%$ CL exclusion cross sections from ATLAS [20] are given as red dots, connected by solid red lines. These lines do not correspond to true experimental analyses and are only indicative. Also shown as dotted red line is the projection for a combination of ATLAS and CMS data (see the end of the previous section and the discussion below).

The crossing point between the black and the red line corresponds to the highest $m_{\tilde{\chi}_{2}^{0}}$ value that is excluded by ATLAS, see Ref. [20]. However, taking into account the decay $\tilde{\chi}_{2}^{0} \rightarrow \tilde{\chi}_{1}^{0} h_{1}$ as well as a variation of the sign of $M_{1}$, resulting in the green and blue lines, moves the highest excluded $m_{\tilde{\chi}_{2}^{0}}$ to substantially smaller values. In the case of $m_{\tilde{\chi}_{1}^{0}}=0$, as shown in the upper left plot in Fig. [3, the exclusion bound for $m_{\tilde{\chi}_{2}^{0}}$ moves from $\sim 310 \mathrm{GeV}$ down to $\sim 140 \mathrm{GeV}$. For positive $M_{1}$ the decay to a Higgs boson is enhanced, resulting in a reduced $\operatorname{BR}\left(\tilde{\chi}_{2}^{0} \rightarrow \tilde{\chi}_{1}^{0} Z\right)$, while a smaller reduction is obtained for negative $M_{1}$. This can be understood from the dependence of the decay amplitudes for $\tilde{\chi}_{2}^{0} \rightarrow \tilde{\chi}_{1}^{0} Z / h_{1}$ as discussed in Sec. 2.2. From those expressions it is clear that the enhancement of the decay to Higgs bosons increases with $M_{1}$. The corresponding strong variation of the partial decay widths leads to a strong variation in $\operatorname{BR}\left(\tilde{\chi}_{2}^{0} \rightarrow \tilde{\chi}_{1}^{0} Z\right)$ Consequently, the differences of the excluded $m_{\tilde{\chi}_{2}^{0}}$ values for opposite signs of $M_{1}$ becomes larger with increasing $m_{\tilde{\chi}_{1}^{0}}$, as is visible comparing the green and the blue curves in the four plots in Fig. 3. The $\mathcal{O}(1 / \tan \beta)$ term in Eq. (13) further increases this ratio of couplings, suppressing the branching ratio to $\tilde{\chi}_{1}^{0} Z$, especially for small $\tan \beta$. We discuss this effect in Sec. 4.2, where we compare the limits for $\tan \beta=6$ (Fig. (3)) and $\tan \beta=20$ (Fig. (4).

A similar conclusion holds for the projected combination of ATLAS and CMS results, shown as red-dotted lines. The reduced statistical error leads to an increase of the excluded values of $m_{\tilde{\chi}_{2}^{0}}$ by $\mathcal{O}(10 \mathrm{GeV})$ for $m_{\tilde{\chi}_{1}^{0}}=0,50 \mathrm{GeV}$. For $m_{\tilde{\chi}_{1}^{0}}=100 \mathrm{GeV}$, where the current analysis just barely sets a limit, the exclusion is larger, while for $m_{\tilde{\chi}_{1}^{0}}=150 \mathrm{GeV}$ neither the current nor the combined analysis yield any exclusion limit.

As explained in Sec. 2.2 a change in the phase of $M_{1}$ (here from 0 to $\pi$ ) does not only change the couplings but also has a dynamical effect on the decay processes, which depends on the relative $\mathcal{C P}$ of the two neutralinos and the Higgs or gauge boson. The corresponding p-wave suppressed (s-wave) amplitude for opposite (equal) relative $\mathcal{C P}$ of the neutralinos and the Higgs or $Z$ boson is most pronounced at the corresponding neutralino decay thresholds, while it becomes negligible for boosted Higgs bosons or gauge boson: 7 . The p-wave suppression effect in the $M_{1}<0$ scenario, compared to the s-wave $M_{1}>0$ decays is reflected in the softer rise of the green curve at the threshold for the decay to the Higgs boson. Since both the $Z$ and the lightest Higgs boson are $\mathcal{C} \mathcal{P}$-even 8 , the effect cancels out in the branching ratio for larger mass differences. Notice, however, that this will not be the case if there are additional decay channels open, as will be discussed in Sec. 4.4 for the DM-motivated

\footnotetext{
${ }^{6}$ In Ref. [15] it was pointed out that, in the large $\mu$ regime, the gaugino pair production process with subsequent decay of a neutralino to a Higgs boson and the LSP would have the highest reach sensitivity for very large luminosities at LHC14. Notice that, for $\left|M_{1}\right| \simeq M_{2}$, the phase of $M_{1}$ could have a significant effect on this reach.

${ }^{7}$ Analyses for neutralino decays to boosted Higgs bosons have been presented in [15, 17, 60.

${ }^{8}$ In case of a complex $M_{1}$ the lightest Higgs will receive a very small $\mathcal{C} \mathcal{P}$-odd admixture.
} 
scenario $S^{\mathrm{DM}}$.

We also briefly investigate the effects of a variation of the overall sfermion mass scale, $M_{\text {SUSY }}$, i.e. the $S_{\text {ATLAS }}^{\text {SUSY }}$ scenario. In the upper left plot of Fig. 3, besides the production cross section using the default value $M_{\mathrm{SUSY}}\left(=M_{\tilde{q}_{1,2}}=M_{\tilde{q}_{3}}=M_{\tilde{\ell}}\right)=2 \mathrm{TeV}$ shown as a black line, we also show as dotted (dot-dashed) gray line the $\tilde{\chi}_{1}^{ \pm} \tilde{\chi}_{2}^{0}$ production cross section for $M_{\tilde{q}_{1,2}}=$ 1.2(3) TeV. Choosing the squarks of the first two families at (roughly) their experimental lower mass limit increases the destructive interference of the $t$-channel squark exchange with the $s$-channel gauge boson exchange production channels. The effect is negligible for the smaller neutralino/chargino masses where the $s$-channel dominates, while it results in a suppression of over $30 \%$ for the largest masses shown. Accordingly, we observe a smaller (larger) production cross section for a smaller (larger) value of $M_{\tilde{q}_{1,2}}$. While the effects are small in comparison to taking into account the decay to Higgs bosons (green line), one can observe that the low $M_{\tilde{q}_{1,2}}$ value has an effect as sizable as the combination of ATLAS and CMS data. We will not investigate the effects of a variation of $M_{\tilde{q}_{1,2}}$ further.

\section{$4.2 \tan \beta$ dependence}

As the change in $\tan \beta$ has a negligible effect on the production cross section, by definition the ATLAS limits do not depend on $\tan \beta$. However, as seen in Eqs. (8), the couplings of the neutralinos to the Higgs bosons are strongly affected by $\tan \beta$, resulting in a larger branching ratio of the second neutralino to a $Z$ boson and the LSP. The experimentally excluded region changes accordingly, with chargino and second lightest neutralino masses excluded up to higher masses than for $\tan \beta=6$. This is illustrated in Fig. 4, where we show the production cross section times the branching ratios for the same parameters as in Fig. 3 except for $\tan \beta$, which is increased from 6 to 20, i.e. for scenario $S_{\text {ATLAS }}^{\tan \beta}$. The mass exclusion limits lie at $190 \mathrm{GeV}$ and $200 \mathrm{GeV}$ for a massless LSP, for the tree-level and NLO results, respectively.

For an LSP of $50 \mathrm{GeV}$ the exclusion limits are found to be very close to the threshold for Higgs decay for positive $M_{1}$ and between $230 \mathrm{GeV}$ and $250 \mathrm{GeV}$ for negative $M_{1}$. For LSP masses above $\sim 100 \mathrm{GeV}$ no chargino masses are excluded once the decay of the second lightest neutralino to the Higgs boson is open. In this region a more meaningful quantity to consider is the ratio of the excluded cross section divided by the theoretical production cross section times branching ratios, indicating the required "improvement" necessary for an exclusion. For instance, for $m_{\tilde{\chi}_{1}^{0}}=100 \mathrm{GeV}, m_{\tilde{\chi}_{2}^{0}}=250 \mathrm{GeV}$ and $\tan \beta=6$ this ratio is, respectively, 17.7 and 2.1 for $M_{1}$ positive and negative. For the same masses but for $\tan \beta=20$, this ratio is smaller, 5.1 and 1.1 , respectively.

The results presented in Figs. 3 and 4 are summarized in Fig. 5, where we show the exclusion region in the $m_{\tilde{\chi}_{2}^{0}}-m_{\tilde{\chi}_{1}^{0}}$ plane for $\tan \beta=6$ (upper plot) and $\tan \beta=20$ (lower plot). The solid lines (shaded areas) correspond to the currently analyzed data. The dashed lines are the projection for the combination of ATLAS and CMS LHC8 data, where the exclusion limit is calculated as for the dotted red line in Fig. 3. The red lines show the ATLAS analysis, the green lines take into account the decays $\tilde{\chi}_{2}^{0} \rightarrow \tilde{\chi}_{1}^{0} h_{1}$ for $M_{1}>0$, and the blue ones for $M_{1}<0$. The exclusion curves are not smooth, reflecting the fact that excluded cross sections obtained from ATLAS are only available for a sparse grid of points 

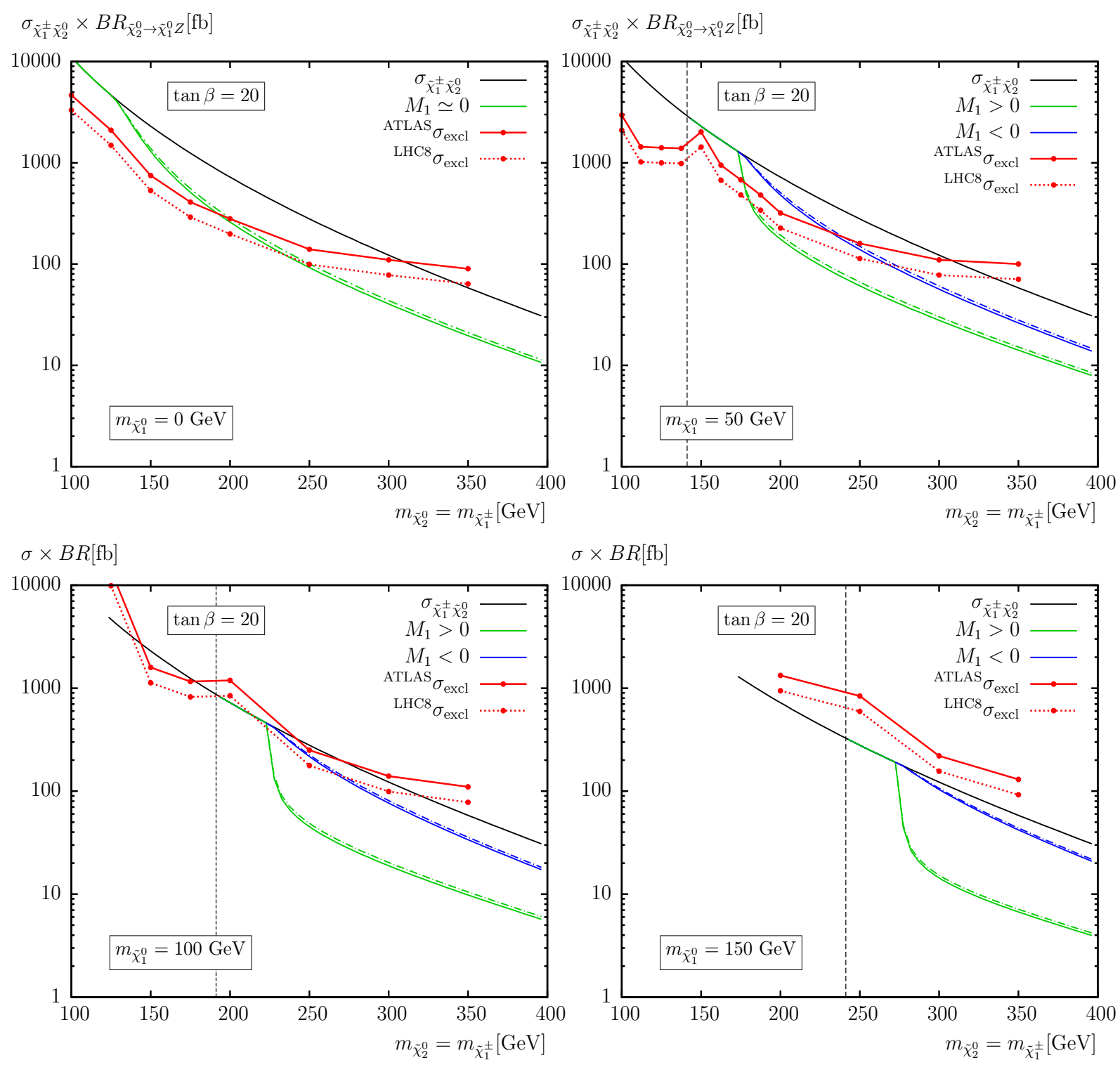

Figure 4: As in Fig. 3, but for $\tan \beta=20$.

in the $m_{\tilde{\chi}_{2}^{0}}-m_{\tilde{\chi}_{1}^{0}}$ plane, and are given by the points in Fig. 3 and 4 where the red lines cross the black, blue and green lines, both for the ATLAS data and for LHC8 combined data. Technically, this is achieved by interpolating the cross section as a function of $m_{\tilde{\chi}_{1}^{0}}$ for fixed values of $m_{\tilde{\chi}_{1}^{0}}$. Note that above the light (dark) gray line the on-shell decay $\tilde{\chi}_{2}^{0} \rightarrow \tilde{\chi}_{1}^{0} Z\left(h_{1}\right)$ is kinematically forbidden. Above the light gray line only off-shell decays of $\tilde{\chi}_{2}^{0}$ are allowed, which is discussed in Sec. 4.8. The results for scenario $S_{\text {ATLAS }}$, i.e. with $\tan \beta=6$, are shown in the upper figure. The dramatic reduction of the excluded area from the ATLAS result in comparison when the decay $\tilde{\chi}_{2}^{0} \rightarrow \tilde{\chi}_{1}^{0} h_{1}$ is taken into account is clearly visible. Only the region where $\tilde{\chi}_{2}^{0} \rightarrow \tilde{\chi}_{1}^{0} h_{1}$ is kinematically forbidden, extended by small strips close to the kinematic limit can be excluded by the current ATLAS analysis. The excluded area grows only marginally taking into account the projection for the LHC8 full data set, i.e. the 

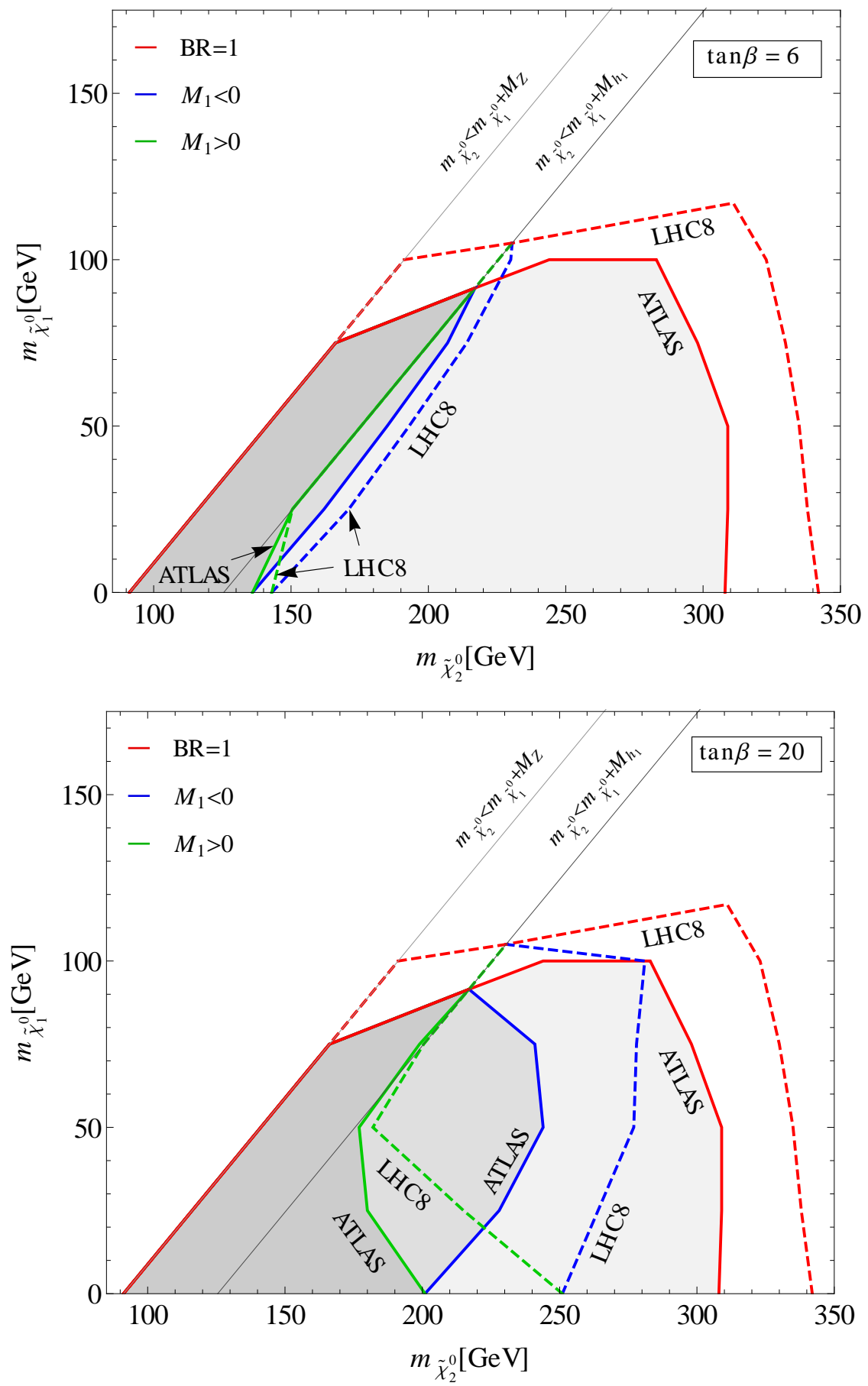

Figure 5: Contours showing the approximate excluded region from $S_{\text {ATLAS }}$ in the $m_{\tilde{\chi}_{2}^{0}}-m_{\tilde{\chi}_{1}^{0}}$ plane with $\tan \beta=6$ (upper plot) and $\tan \beta=20$ (lower plot), The solid lines (shaded areas) correspond to the exclusion for the currently analyzed ATLAS data, and the dashed lines indicate the projection for the combined LHC 8 data, both for the case where it is assumed $\operatorname{BR}\left(\tilde{\chi}_{2}^{0} \rightarrow \tilde{\chi}_{1}^{0} Z\right)=1$, and where the decays $\tilde{\chi}_{2}^{0} \rightarrow \tilde{\chi}_{1}^{0} h_{1}$ are taken into account for $M_{1}>0$ (green), and for $M_{1}<0$ (blue) as indicated, calculating $\operatorname{BR}\left(\tilde{\chi}_{2}^{0} \rightarrow \tilde{\chi}_{1}^{0} Z\right)$ at NLO. Above the light (dark) gray line the on-shell decay $\tilde{\chi}_{2}^{0} \rightarrow \tilde{\chi}_{1}^{0} Z\left(h_{1}\right)$ is kinematically forbidden. 
projected combination of ATLAS and CMS data.

The results for scenario $S_{\mathrm{ATLAS}}^{\tan \beta}$, i.e. with $\tan \beta=20$, are displayed in the lower figure of Fig. 5. While, by definition the curves with $\operatorname{BR}\left(\tilde{\chi}_{2}^{0} \rightarrow \tilde{\chi}_{1}^{0} Z\right)=1$ are identical for $\tan \beta=6$ and $\tan \beta=20$, the regions excluded taking $\tilde{\chi}_{2}^{0} \rightarrow \tilde{\chi}_{1}^{0} h_{1}$ into account are somewhat larger for $\tan \beta=20$. Still a substantial reduction of the excluded regions remains visible. Again, the observations in Fig. [5 can easily be understood in terms of Eq. (8) and (7), where we see that for smaller $\tan \beta$ and large $\mu$ the decay to the Higgs dominates, and the branching ratio to the $Z$ boson substantially smaller than one.

Altogether these results show, on the one hand, how important it is to look at a realistic spectrum (i.e. where the decays to a Higgs boson are not neglected), and on the other hand that dedicated searches for the $W h+E_{T}^{\text {miss }}$ channel are beneficial [15].

\subsection{Complex couplings}

As shown in Sec. 2.2, the partial decay width to Higgs bosons decreases with $\varphi_{M_{1}}$, due to the decrease in the neutralino-Higgs coupling, which from Eq. (8) is seen to be most dependent on the phase for large $\tan \beta$. The $\varphi_{M_{1}}$ dependence could have an interesting impact on the exclusion bounds on $M_{1}$ and $M_{2}$. This is illustrated in Fig. 6, where we interpret the ATLAS exclusion limits in the $\varphi_{M_{1}}-M_{1}$ plane, for $\tan \beta=6$ (left) and $\tan \beta=20$ (right), and for $\Delta:=M_{2}-M_{1}=M_{h_{1}}, 130,150,180 \mathrm{GeV}$ (defining the value of $M_{2}$ in the plots). The values of $\Delta$ here correspond approximately to diagonal lines in the $m_{\tilde{\chi}_{2}^{0}}-m_{\tilde{\chi}_{1}^{0}}$ plane in 5, starting from the Higgs threshold at $\Delta=M_{h_{1}}$. The solid (dotted) lines correspond to the NLO (tree-level) calculation. We also indicate the limits in red, given by the requirement that the EDMs for thallium and mercury, $d_{\mathrm{Tl}}\left(M_{\mathrm{SUSY}}\right)$ and $d_{\mathrm{Hg}}\left(M_{\mathrm{SUSY}}\right)$, calculated using CPsuperH $2.3[5456]^{9}$ for a specific value of $M_{\text {SUSY }}$ within $S_{\text {ATLAS }}^{\text {SUSY }}$, is below the upper limit, i.e. $d_{\mathrm{Tl}}^{\exp }=9.010^{-25} e \mathrm{~cm}(90 \% \mathrm{CL})$ or $d_{\mathrm{Hg}}^{\exp }=3.110^{-29} e \mathrm{~cm}(95 \% \mathrm{CL})$ [52, 53]. We adopt a common mass scale $M_{\mathrm{SUSY}}=M_{\tilde{q}_{1,2}}=M_{\tilde{q}_{3}}=M_{\tilde{\ell}}$, although the EDMs depend mainly on $M_{\tilde{q}_{1,2}}$ and $M_{\tilde{\ell}_{1,2}}$. Note that the predictions rely on atomic or hadronic matrix elements which can have large theoretical uncertainties, ranging from $\sim 10 \%$ to $50 \%$ (see e.g. Ref. [61]). Of the numerous options available in CPSuperH 2.3 for the parametrization of the Schiff moment contribution to $d_{\mathrm{Hg}}$, as described in Ref. [62], we choose the result of Ref. 63]. We display the limit for the EDM that provides the strongest bound, i.e. from $d_{\mathrm{Tl}}$ for $\tan \beta=6$ and from $d_{\mathrm{Hg}}$ for $\tan \beta=20$. Although $M_{\mathrm{SUSY}}=0.8 \mathrm{TeV}$ is disfavored at the LHC, the lines are indicated for comparison, as there is no exclusion from the EDMs for higher values of $M_{\mathrm{SUSY}}$ (i.e. in $S_{\mathrm{ATLAS}}$ ) for the case $\tan \beta=6$.

From Fig. 6 one can clearly see the effect of $\varphi_{M_{1}}$ on the exclusion limit on $M_{1}$, being much higher for $\varphi_{M_{1}}=\pi$ than for $\varphi_{M_{1}}=0$, as also seen in Fig. [5 and discussed in Sec. 4.1, Furthermore, as discussed above, it can be observed that the effect of the phase is much more pronounced for lower values of $\tan \beta$. On changing $\varphi_{M_{1}}$ from 0 to $\pi$, for $\Delta=130 \mathrm{GeV}$, the limit on $M_{1}$ changes by $\sim 80 \mathrm{GeV}$ for $\tan \beta=6$ as opposed to $50 \mathrm{GeV}$ for $\tan \beta=20$. Note that the exclusion disappears completely for $\Delta>135 \mathrm{GeV}$ for $\tan \beta=6$ and $\Delta>200 \mathrm{GeV}$ for $\tan \beta=20$. The $\Delta=M_{h_{1}}$ line is also shown, illustrating that below the Higgs threshold, the dependence on $\varphi_{M_{1}}$ vanishes. Further effects of $\varphi_{M_{1}}$ will be discussed in Sec. 4.4,

\footnotetext{
${ }^{9}$ Similar results can be obtained with FeynHiggs 42,45$]$.
} 

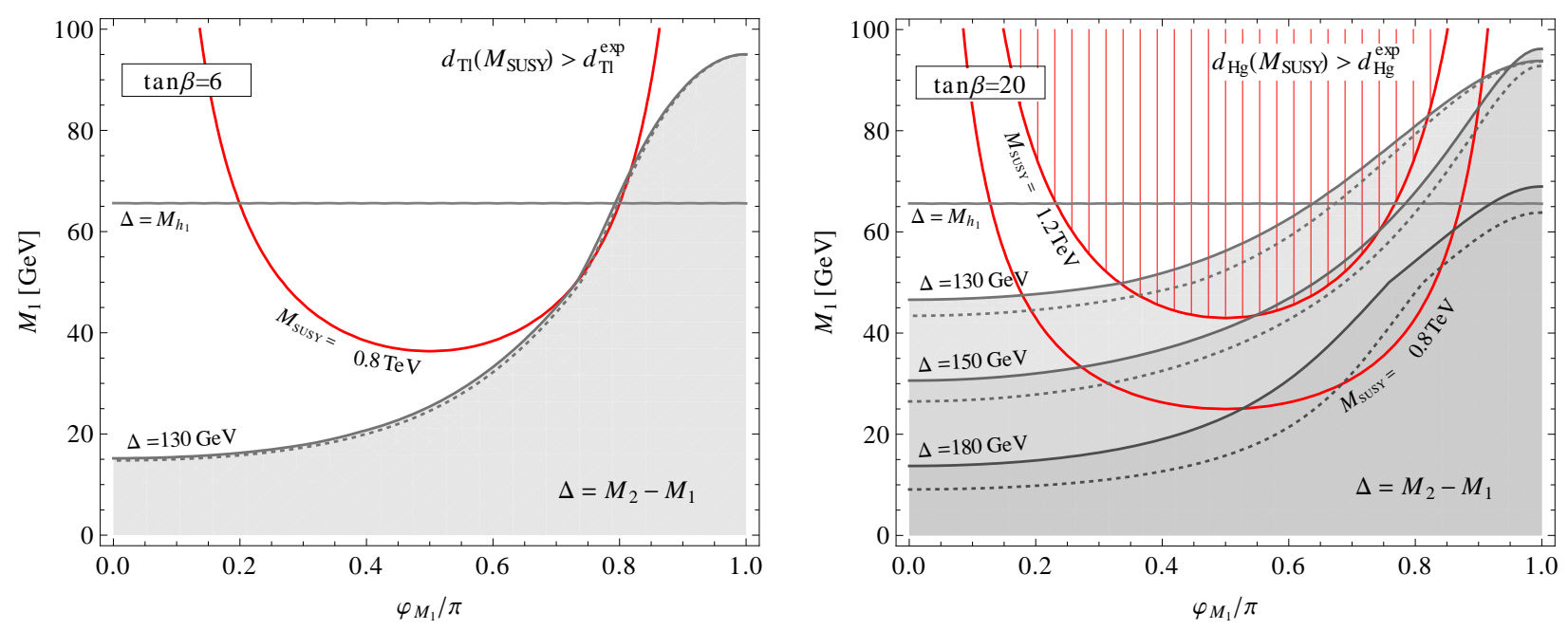

Figure 6: Contours showing the excluded region from currently analyzed ATLAS data for $S_{\text {ATLAS }}$ in the $M_{1}-\varphi_{M_{1}}$ plane, with $\tan \beta=6$ (left) and $\tan \beta=20$ (right). $M_{2}$ is fixed via $\Delta=M_{2}-M_{1}$, which corresponds approximately to diagonal lines in e.g. Fig. 5 , parallel to the Higgs threshold given by $\Delta=M_{h_{1}}$. The solid (dotted) lines indicate that the exclusion contours are calculated using NLO (tree-level) branching ratios for the $\tilde{\chi}_{2}^{0}$ decays. At $\Delta=150 \mathrm{GeV}$ for $\tan \beta=6$ and $\Delta=210 \mathrm{GeV}$ for $\tan \beta=20$ there is no exclusion from ATLAS. The red lines define exclusion contours for $S_{\text {ATLAS }}^{\text {SUSY }}$, where $M_{\text {SUSY }}$ is indicated (see text), from the EDMs of thallium $\left(d_{\mathrm{Tl}}\right)$ and mercury $\left(d_{\mathrm{Hg}}\right)$.

The relevance of the one-loop corrections, i.e. the difference between solid and dotted lines, is clearly visible for $\tan \beta=20$, in the right plot of Fig. 6, via the shift in the excluded $M_{1}$ value (for fixed $\varphi_{M_{1}}$ ), which ranges from $\sim 0$ for $\Delta=M_{h_{1}}$ and $\sim 12 \mathrm{GeV}$ for $\Delta=$ $180 \mathrm{GeV}$. A more detailed discussion of the impact of NLO corrections can be found in Sec. 4.6.

\subsection{The DM scenario: effect of a low scalar tau mass}

In this section we briefly analyze the effect of making the low scalar tau nearly degenerate with the LSP, i.e. scenario $S^{\mathrm{DM}}$. In this way the $\tilde{\chi}_{1}^{0}$ provides the correct amount of relic Cold Dark Matter [3, 57]. In this scenario the new $\tilde{\chi}_{2}^{0}$ decay channel, $\tilde{\chi}_{2}^{0} \rightarrow \tilde{\tau}_{1} \tau$ opens up. The appearance of this decay channel not only reduces the $\operatorname{BR}\left(\tilde{\chi}_{2}^{0} \rightarrow \tilde{\chi}_{1}^{0} Z\right)$, i.e. the channel taken into account by the ATLAS analysis, but also results in a new channel to be analyzed, as in Ref. [8, 9, 18, 20, 22, A combination of the experimental analyses for these two channels is clearly beyond the scope of our paper. Consequently in this scenario, we analyze the branching ratios, but do not attempt to re-evaluate the exclusion limits in the $m_{\tilde{\chi}_{2}^{0}}-m_{\tilde{\chi}_{1}^{0}}$ plane. The problem of combination of analyses due to the appearance of new channels becomes even more important in this scenario, since the channel $\tilde{\chi}_{1}^{ \pm} \rightarrow \tilde{\tau}_{1} \nu_{\tau}$ also opens up, which can have a non-negligible branching ratio [33]. However, we will not discuss this point further here.

The decays of gauginos to a lepton-slepton pair strongly depends on the character of 

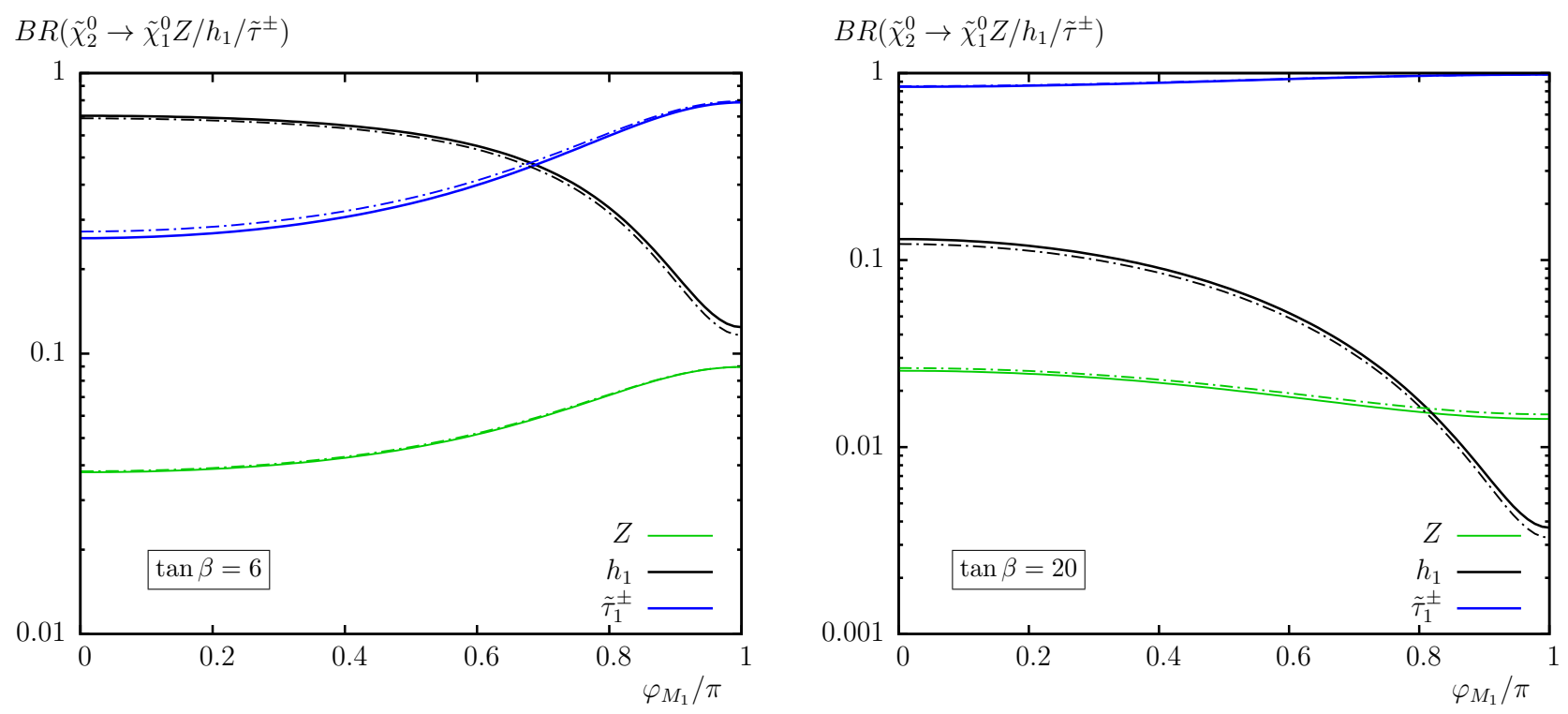

Figure 7: $\operatorname{BR}\left(\tilde{\chi}_{2}^{0} \rightarrow \tilde{\chi}_{1}^{0} Z\right), \operatorname{BR}\left(\tilde{\chi}_{2}^{0} \rightarrow \tilde{\chi}_{1}^{0} h_{1}\right)$ and $\operatorname{BR}\left(\tilde{\chi}_{2}^{0} \rightarrow \tilde{\tau}_{1} \tau\right)$ in the $S^{\mathrm{DM}}$ scenario as a function of $\varphi_{M_{1}}$ for $\tan \beta=6$ (left) and 20 (right). Solid lines show the tree-level result, whereas dash-dotted lines display the NLO branching ratios.

the sleptons: while the decay to left-handed sleptons is unsuppressed, that to right-handed sleptons is proportional to the Yukawa coupling, which is strongly suppressed by the small mass of the leptons. As here we are interested in the interplay of the $Z, h_{1}$ and $\tilde{\tau}_{1}$ channels, we focus on the region of parameter space where the suppressed neutralino decays to $Z$ and Higgs bosons (see Sec. 2.2) are competitive with the decay to $\tilde{\tau}_{1} \tau$. Unsuppressed decays to a left-handed $\tilde{\tau}$ and a $\tau$ would strongly dominate all other decay channels and are thus of limited interest here. Decays to right-handed staus, on the other hand, are potentially interesting from a phenomenological point of view, and we consider the possibility that the right-handed soft SUSY-breaking parameter $M_{\tilde{\tau}_{R}}$ is much smaller than the left-handed one, resulting in an almost purely right-handed lightest stau, as given in the definition of $S^{\mathrm{DM}}$. The decay to a pure $\tilde{\tau}_{R}$ results in the minimum possible $\mathrm{BR}$ to $\tilde{\tau}_{1} \tau$ of the gaugino-like neutralino. However, the stau mixing induced by the non-diagonal entry in the stau mass matrix, given by $m_{\tau} \times \mu \tan \beta$ (using $A_{\tau}=0$ ), adds a small $\tilde{\tau}_{L}$ admixture, strongly enhancing the decay $\tilde{\chi}_{2}^{0} \rightarrow \tilde{\tau}_{1} \tau$. We find a stau mixing angle of $\theta_{\tilde{\tau}} \simeq 0.25 \times 10^{-3}$ for $\tan \beta=6$ and $\theta_{\tilde{\tau}} \simeq 0.7 \times 10^{-3}$ for $\tan \beta=20$. Both the increased left-handed component, as well as the $\tan \beta$-dependence of the Yukawa couplings enhance decay $\tilde{\chi}_{2}^{0} \rightarrow \tilde{\tau}_{1} \tau$ with growing $\tan \beta$.

The results are shown in Fig. 7, where we display $\operatorname{BR}\left(\tilde{\chi}_{2}^{0} \rightarrow \tilde{\chi}_{1}^{0} Z\right), \operatorname{BR}\left(\tilde{\chi}_{2}^{0} \rightarrow \tilde{\chi}_{1}^{0} h_{1}\right)$ and $\operatorname{BR}\left(\tilde{\chi}_{2}^{0} \rightarrow \tilde{\tau}_{1}^{ \pm} \tau^{\mp}\right)$ as a function of $\varphi_{M_{1}}$ for $\tan \beta=6$ and 20 in the left and right plot, respectively. Solid lines show the tree-level result, whereas dash-dotted lines display the NLO branching ratios. We see that the branching ratio for the decay to $\tilde{\chi}_{1}^{0} Z$ is reduced to the percent level, in general lying between $\sim 1.5$ and $\sim 3 \%$ and reaching at most $\sim 9 \%$ for $\tan \beta=6$ and $\varphi_{M_{1}}=\pi$. This strong reduction would make this decay challenging at the LHC13/LHC14. Note that in our benchmark scenarios with a bino-like LSP, the decay of the second lightest neutralino to a lepton-slepton pair has a negligible dependence on $\varphi_{M_{1}}$, 
and the increase of $\operatorname{BR}\left(\tilde{\chi}_{2}^{0} \rightarrow \tilde{\tau}_{1} \tau\right)$ with $\varphi_{M_{1}}$ is due to the decrease in the partial decay widths to $Z$ and Higgs bosons, as discussed in Sec. 2.2. There we observed that these couplings are largest when $\varphi_{M_{1}}=0$, see Eq. (8), and that the dependence on the phase is largest for large $\tan \beta$, in agreement with Fig. 7 .

\subsection{Gaugino vs. higgsino production: the low $\mu$ case}

The limits on searches for electroweak SUSY particles presented by ATLAS and CMS assume that the relevant particles are gaugino-like, corresponding to a relatively large value of $\mu$. In this region of the SUSY parameter space the production cross sections for $\tilde{\chi}_{1}^{ \pm} \tilde{\chi}_{2}^{0}$ are largest due to the unsuppressed coupling to the $W$-boson. On the other hand, when $M_{1}<\mu<M_{2}$ the second and third neutralino, as well as the lightest chargino, are higgsino-like and roughly degenerate in mass, changing the phenomenology of the particles under investigation. In this section we briefly analyze the phenomenology in the scenario $S_{\text {low }-\mu}$ with $M_{1}<\mu<M_{2}$ with $\mu=100 \ldots 400 \mathrm{GeV}$ and $M_{2}=500 \mathrm{GeV}$.

In Fig. 8 we show the results obtained in $S_{\text {low }-\mu}$, in analogy to Fig. 3 , for $\tan \beta=6$ and for $m_{\tilde{\chi}_{1}^{0}}=0,50,100 \mathrm{GeV}$ in the top, middle and lower row, respectively. Contrary to Fig. 3, besides the results for $\tilde{\chi}_{1}^{ \pm} \tilde{\chi}_{2}^{0}$ production shown in the left column as function of $m_{\tilde{\chi}_{2}^{0}}$, we present in the right column the analysis for $\tilde{\chi}_{1}^{ \pm} \tilde{\chi}_{3}^{0}$ production as a function of $m_{\tilde{\chi}_{3}^{0}}$. As discussed above, in this scenario we have $m_{\tilde{\chi}_{1}^{ \pm}} \approx m_{\tilde{\chi}_{2}^{0}} \approx m_{\tilde{\chi}_{3}^{0}}$. The mass differences range from a few to a few tenths of $\mathrm{GeV}$, not allowing a clean separation of these channels at the LHC. This spectrum also forbids two-body decays between these states. A full experimental analysis will combine the two production channels. However, such a combination of different channels goes beyond the scope of our paper, and we discuss the mass limits obtained for the two production channels individually.

As a first general observation it should be noted that the smaller couplings to gauge bosons suppress the production cross section by almost a factor of 4 for low chargino masses to $\sim 2$ for high masses (where a substantial mixing with gauginos increases the coupling to the $W$-boson). Notice that this suppression could be partially overcome by a combination of the two production cross sections. In order to interpret the ATLAS limits [20] in this scenario we must take into account that the chargino is not exactly degenerate with the two neutralinos. We interpret the limits as a function of the corresponding neutralino mass, which is larger than that of the chargino. In our simple interpretation of the ATLAS limits, as shown in Fig. 8, no mass value for $m_{\tilde{\chi}_{2}^{0}}$ or $m_{\tilde{\chi}_{3}^{0}}$ can be excluded. Only the combination of ATLAS and CMS, shown as red-dotted line could yield an exclusion for $m_{\tilde{\chi}_{1}^{0}}=0$ for $m_{\tilde{\chi}_{3}^{0}} \lesssim 200 \mathrm{GeV}$.

The most interesting observation in this scenario is the complementarity between $\tilde{\chi}_{1}^{ \pm} \tilde{\chi}_{2}^{0}$ and $\tilde{\chi}_{1}^{ \pm} \tilde{\chi}_{3}^{0}$ production. For $M_{1}$ positive, shown as the green lines, only $\operatorname{BR}\left(\tilde{\chi}_{2}^{0} \rightarrow \tilde{\chi}_{1}^{0} Z\right)$ shows a strong suppression, whereas $\operatorname{BR}\left(\tilde{\chi}_{3}^{0} \rightarrow \tilde{\chi}_{1}^{0} Z\right)$ is close to one, as noticed in Ref. [64]. This complementary behavior is due largely to the Higgs-higgsino-gaugino couplings discussed in Sec. 2.2 and the fact that $\tilde{\chi}_{2}^{0}$ and $\tilde{\chi}_{3}^{0}$ are opposite in their $\mathcal{C P}$ behavior (being the SUSY partners of the $\mathcal{C P}$-even and -odd Higgses). This is also reflected in the difference between $M_{1}$ positive or negative. While for $\tilde{\chi}_{1}^{ \pm} \tilde{\chi}_{2}^{0}$ a negative $M_{1}$ results in a larger $\operatorname{BR}\left(\tilde{\chi}_{2}^{0} \rightarrow \tilde{\chi}_{1}^{0} Z\right)$, it is exactly opposite for $\tilde{\chi}_{1}^{ \pm} \tilde{\chi}_{3}^{0}$, where only for $M_{1}$ negative a strong reduction of $\operatorname{BR}\left(\tilde{\chi}_{3}^{0} \rightarrow \tilde{\chi}_{2}^{0} Z\right)$ is found. Consequently, for $M_{1}<0$ both channels, $\tilde{\chi}_{1}^{ \pm} \tilde{\chi}_{2}^{0}$ and $\tilde{\chi}_{1}^{ \pm} \tilde{\chi}_{3}^{0}$ are suppressed. 

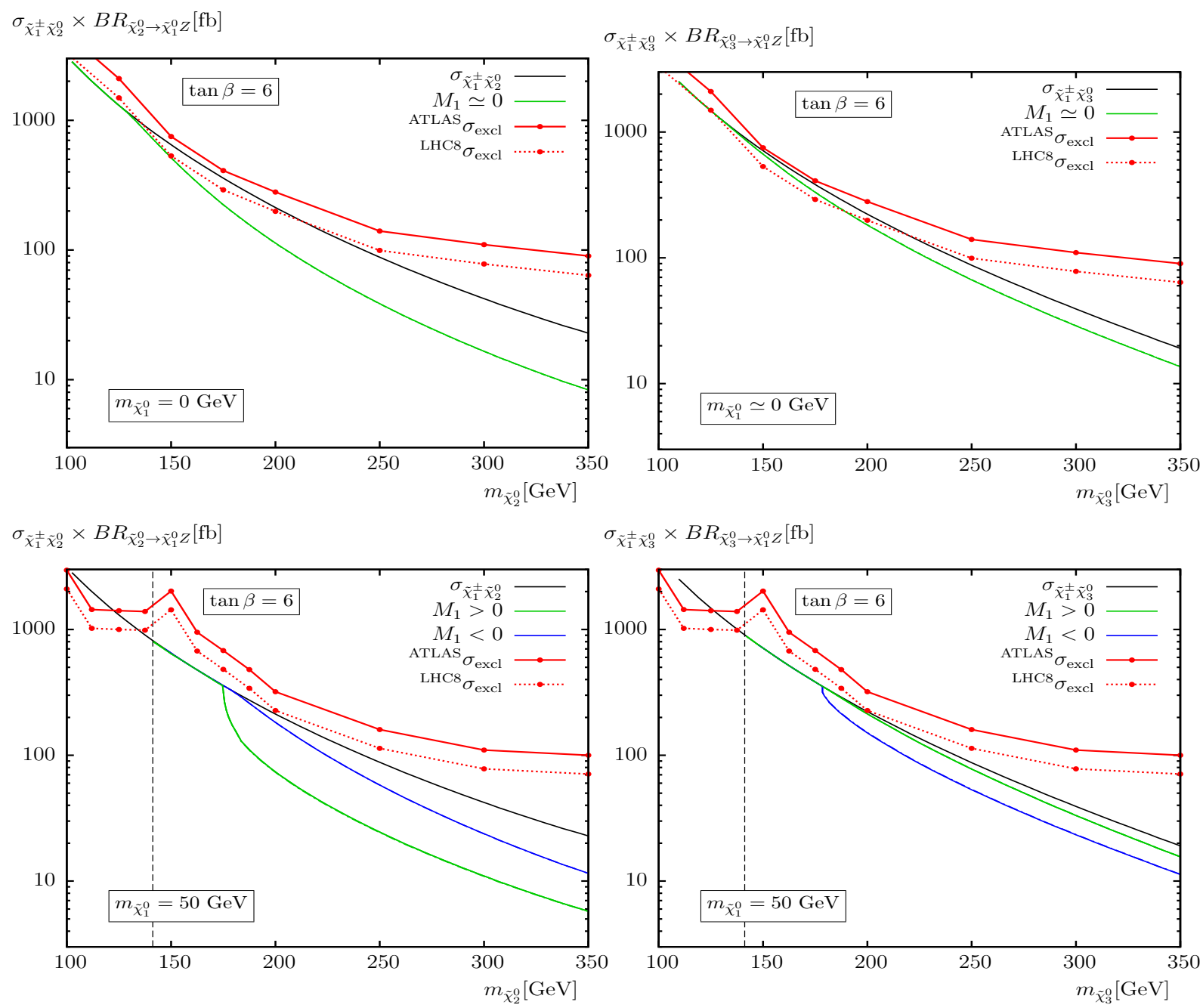

$\sigma_{\tilde{\chi}_{1}^{ \pm} \tilde{\chi}_{3}^{0}} \times B R_{\tilde{\chi}_{3}^{0} \rightarrow \tilde{\chi}_{1}^{0} Z}[\mathrm{fb}]$
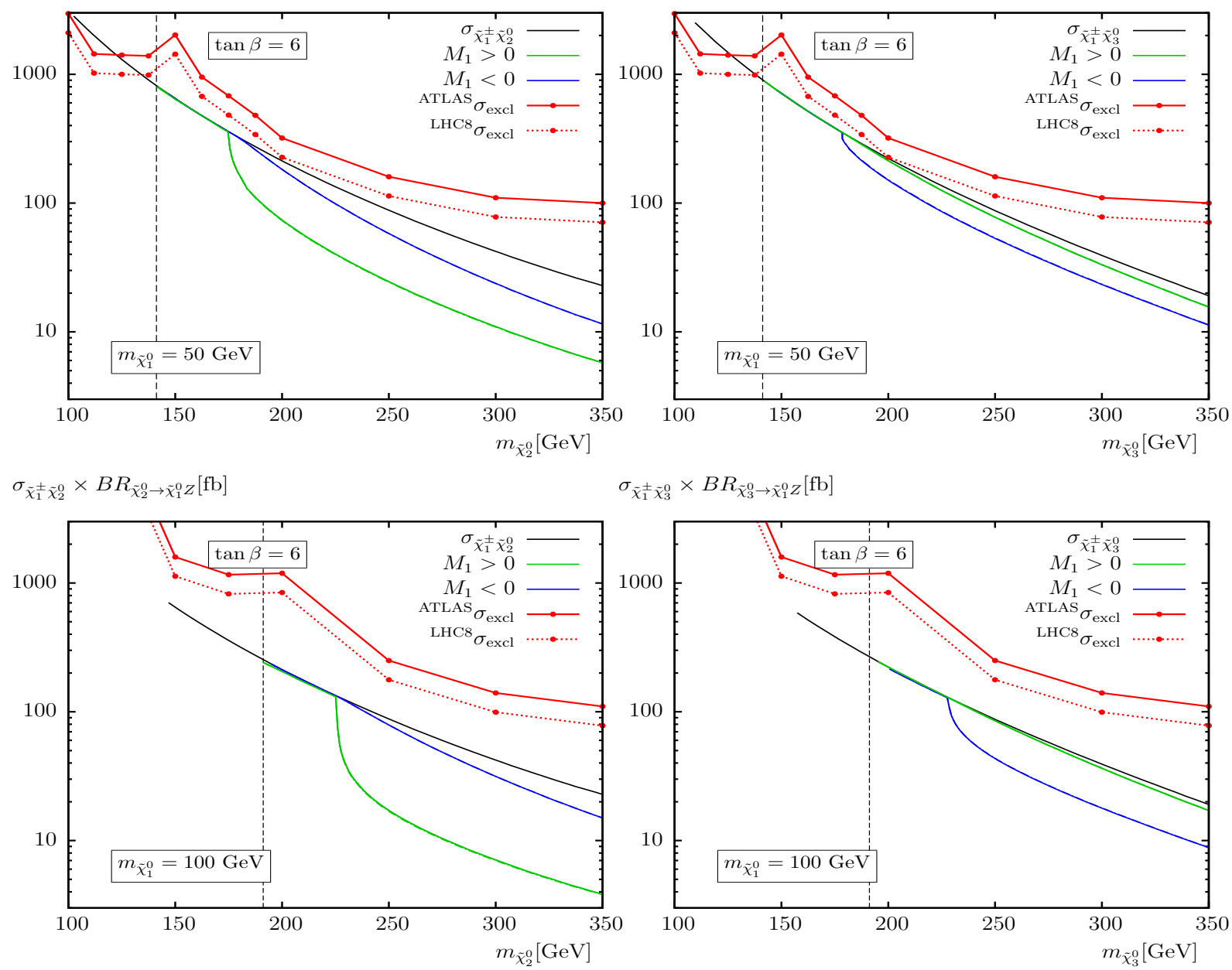

$\sigma_{\tilde{\chi}_{1}^{ \pm} \tilde{\chi}_{3}^{0}} \times B R_{\tilde{\chi}_{3}^{0} \rightarrow \tilde{\chi}_{1}^{0} Z}[\mathrm{fb}]$

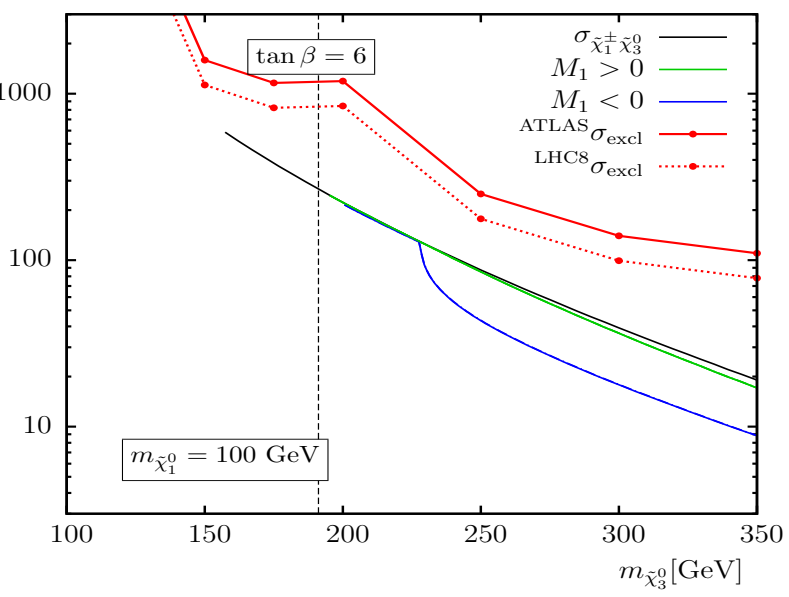

Figure 8: As in Fig. 3, but for $\tilde{\chi}_{1}^{ \pm} \tilde{\chi}_{2}^{0}$ (left) and $\tilde{\chi}_{1}^{ \pm} \tilde{\chi}_{3}^{0}$ (right) in scenario $S_{\text {low }-\mu}$, with $M_{1}$ is chosen such that $m_{\tilde{\chi}_{1}^{0}}=0,50,100 \mathrm{GeV}$, in the top, middle and bottom plot, respectively. 
In summary: the case of a low $\mu$ parameter appears to be more challenging for ATLAS and CMS than the case of a large $\mathrm{TeV}$-scale $\mu$. The production cross sections are suppressed for small $\mu$, and in particular for negative $M_{1}$ the $\operatorname{BR}\left(\tilde{\chi}_{2}^{0} / \tilde{\chi}_{3}^{0} \rightarrow \tilde{\chi}_{1}^{0} Z\right)$ lead to a further suppression and hardly any limit can be derived from the 2012 data. Only a combination of the two search channels might partly overcome this reduction.

\subsection{Impact of radiative corrections}

It is of interest to calculate the predicted cross sections and the resulting exclusion bounds at NLO (the full one-loop level), in order to assess if there are regions of parameter space where these are large or of importance and must be taken into account. A detailed study of the NLO corrections to neutralino decays was carried out for the complex MSSM in Ref. [34]. There it was found that the size of the corrections for $\tilde{\chi}_{i}^{0} \rightarrow \tilde{\chi}_{1}^{0} Z$ and $\tilde{\chi}_{i}^{0} \rightarrow \tilde{\chi}_{1}^{0} h_{1}$ were greater in general, when the decaying neutralino was higgsino-like rather than wino-like (assuming that $\tilde{\chi}_{1}^{0}$ is predominantly bino-like). However, in the case of the decaying neutralino being wino-like, the radiative corrections were strongly dependent on $\varphi_{M_{1}}$. This arises when the tree-level decay is suppressed, due to the coupling or due to a p-wave suppression as described in Sec. 4.3, whereas the radiative corrections may not be suppressed by these mechanisms, particularly in mixed scenarios. In such cases at different values of $\varphi_{M_{1}}$, the tree-level decay is no longer suppressed, and therefore the relative size of the NLO contribution is smaller. For $S_{\text {ATLAS }}$ the situation is different, as $\mu$ is at the $\mathrm{TeV}$ scale, and these effects are not so pronounced. In Tab. 2, we have summarized the percentage contribution of the loop corrections for the decay $\tilde{\chi}_{2}^{0} \rightarrow \tilde{\chi}_{1}^{0} Z$ for representative parameter points of each of the scenarios defined in Tab. 1. Here we use

$$
\Delta \Gamma^{\text {loop }}:=\frac{\Gamma^{\mathrm{NLO}}-\Gamma^{\text {tree }}}{\Gamma^{\text {tree }}}, \quad \Delta \mathrm{BR}^{\text {loop }}:=\frac{\mathrm{BR}^{\mathrm{NLO}}-\mathrm{BR}^{\text {tree }}}{\mathrm{BR}^{\text {tree }}} .
$$

One can observe the following:

- Our baseline scenario, motivated by the ATLAS analysis, has a high $\mu$ and $M_{\mathrm{SUSY}}$ as well as small $\tan \beta$, resulting in a change in the branching ratio to $Z$ bosons of the order of $8 \%$. As seen from the relative correction to $\Gamma\left(\tilde{\chi}_{2}^{0} \rightarrow \tilde{\chi}_{1}^{0} Z\right)$, small due to a cancellation between the chargino/neutralino and sfermion loops, this $8 \%$ arises due to the corrections to the decay width $\Gamma\left(\tilde{\chi}_{2}^{0} \rightarrow \tilde{\chi}_{1}^{0} h_{1}\right)$.

- As seen in Eq. (8) $) \Gamma\left(\tilde{\chi}_{2}^{0} \rightarrow \tilde{\chi}_{1}^{0} h_{1}\right)$ is maximum at $\varphi_{M_{1}}=0$, where the loop corrections amount to $-11 \%$, and reaches a minimum when the coupling is smallest at $\varphi_{M_{1}}=\pi$, where the corrections are maximal, i.e. $-15 \%$.

- On decreasing $M_{\mathrm{SUSY}}$, the correction to the branching ratio increases by $50 \%$, as the correction to $\Gamma\left(\tilde{\chi}_{2}^{0} \rightarrow \tilde{\chi}_{1}^{0} Z\right)$ increases, due to the sfermion loop contribution, to $-4 \%$ at $\varphi_{M_{1}}=0$ and $-2 \%$ at $\varphi_{M_{1}}=\pi$. There is also a large increase in the magnitude of the loop corrections to $\Gamma\left(\tilde{\chi}_{2}^{0} \rightarrow \tilde{\chi}_{1}^{0} h_{1}\right)$ from $\sim-18 \%$ at $\varphi_{M_{1}}=0$ to $-25 \%$ at $\varphi_{M_{1}}=\pi$. Overall corrections to $\operatorname{BR}\left(\tilde{\chi}_{2}^{0} \rightarrow \tilde{\chi}_{1}^{0} Z\right)$ larger than $10 \%$ can be observed.

- Finally, the effect of $\mu$ was investigated in both scenarios $S_{\text {ATLAS }}^{\mu}$ and $S_{\text {low- }-\mu}$, and found to play an important role. As $\mu$ increases, as in $S_{\text {ATLAS }}^{\mu}$, the chargino/neutralino 
contribution to the corrections decouples. Therefore the cancellation seen for $S_{\text {ATLAS }}$ no longer exists, and the correction to the decay width (mostly due to sfermion loops) becomes $-5 \%$. This means that the corrections to $\Gamma\left(\tilde{\chi}_{2}^{0} \rightarrow \tilde{\chi}_{1}^{0} Z\right)$ and $\Gamma\left(\tilde{\chi}_{2}^{0} \rightarrow \tilde{\chi}_{1}^{0} h_{1}\right)$ partially compensate each other. For low $\mu\left(S_{\text {low }-\mu}\right)$, the chargino/neutralino loop contribution increases, resulting in the effect on the BR canceling out nearly completely and is found to be at the level of $-1 \%$.

Note that the impact of loop corrections at large $\tan \beta$ is nicely illustrated in Fig. 6, where the difference between the tree-level and NLO exclusion line reaches up to $\sim 12 \mathrm{GeV}$, resulting in a $30 \%$ change in the excluded $M_{1}$. Therefore the NLO corrections can have a substantial effect and should eventually be included when interpreting exclusion limits (or discovery results) for MSSM parameters.

\begin{tabular}{|c||c|c|c|c|c|c|c|c|c|}
\hline Scenario & $\left|M_{1}\right|$ & $M_{2}$ & $\varphi_{M_{1}}$ & $\mu$ & $\tan \beta$ & $M_{\text {SUSY }}$ & $M_{\tilde{\tau}_{R}}$ & $\Delta \mathrm{BR}^{\text {loop }}$ & $\Delta \Gamma^{\text {loop }}$ \\
\hline \hline$S_{\text {ATLAS }}$ & 100 & 250 & 0 & 1000 & 6 & 2000 & $M_{\text {SUSY }}$ & $8 \%$ & $<1 \%$ \\
$S_{\text {ATLAS }}$ & 100 & 250 & $\pi$ & 1000 & 6 & 2000 & $M_{\text {SUSY }}$ & $4 \%$ & $1 \%$ \\
\hline$S_{\text {ATLAS }}^{\varphi_{M_{1}}}$ & 100 & 250 & $\pi / 2$ & 1000 & 6 & 2000 & $M_{\text {SUSY }}$ & $8 \%$ & $<1 \%$ \\
\hline$S_{\text {ATLAS }}^{\tan \beta}$ & 100 & 250 & 0 & 1000 & 20 & 2000 & $M_{\text {SUSY }}$ & $8 \%$ & $<1 \%$ \\
$S_{\text {ATLAS }}^{\text {tan } \beta}$ & 100 & 250 & $\pi$ & 1000 & 20 & 2000 & $M_{\text {SUSY }}$ & $4 \%$ & $1 \%$ \\
\hline$S_{\text {ATLAS }}^{\mu}$ & 100 & 250 & 0 & 2000 & 6 & 2000 & $M_{\text {SUSY }}$ & $7 \%$ & $-5 \%$ \\
\hline$S_{\text {ATLAS }}^{\text {SUSY }}$ & 100 & 250 & 0 & 1000 & 6 & 1200 & $M_{\text {SUSY }}$ & $12 \%$ & $-4 \%$ \\
$S_{\text {ATLAS }}^{\text {SUSY }}$ & 100 & 250 & $\pi$ & 1000 & 6 & 1200 & $M_{\text {SUSY }}$ & $11 \%$ & $-2 \%$ \\
\hline \hline$S^{\text {DM }}$ & 100 & 250 & 0 & 1000 & 6 & 2000 & $\left|M_{1}\right|$ & $5 \%$ & $-1 \%$ \\
$S^{\text {DM }}$ & 100 & 250 & $\pi$ & 1000 & 6 & 2000 & $\left|M_{1}\right|$ & $5 \%$ & $-1 \%$ \\
\hline$S_{\text {low }-\mu}$ & 100 & 500 & 0 & 250 & 6 & 2000 & $M_{\text {SUSY }}$ & $-1 \%$ & $2 \%$ \\
$S_{\text {low }-\mu}$ & 100 & 500 & 0 & 350 & 6 & 2000 & $M_{\text {SUSY }}$ & $-1 \%$ & $4 \%$ \\
\hline
\end{tabular}

Table 2: Percentage contribution of the one-loop corrections to the branching ratio and decay width for $\tilde{\chi}_{2}^{0} \rightarrow \tilde{\chi}_{1}^{0} Z$, for each of the scenarios defined in Tab. 1. For those scenario defined in terms of a range in a particular parameter, we specify the value of this parameter used given, with the exception of $\tan \beta$, in $\mathrm{GeV}$. 


\subsection{LHC13 expected sensitivity}

In this section we make a simple projection of the LHC8 results to the future upgrade to the LHC13 assuming a luminosity $\mathcal{L}_{\mathrm{LHC} 13}=100 \mathrm{fb}^{-1}$. In order to estimate the LHC reach without making a full dedicated analysis we need to make an extrapolation of the relevant background. The main irreducible background for the chargino/neutralino direct production with subsequent decays to gauge bosons is diboson production, see e.g. [18,20]. We therefore rescale the number of background events with

$$
R_{\mathrm{bkg}}=\frac{\sigma_{W Z}(13 \mathrm{TeV})}{\sigma_{W Z}(8 \mathrm{TeV})} \times \frac{\mathcal{L}_{\mathrm{LHC} 13}}{\mathcal{L}_{\mathrm{LHC} 8}} .
$$

where $\sigma_{W Z}(x \mathrm{TeV})$ denotes the inclusive $W^{ \pm} Z$ production cross section at LHCx. This projection neglects any effects from the different environment at the higher energy LHC run, for instance the larger pile up. Notice that similar assumptions have been made for projections for scalar top searches at CMS [65], where the main background is related to the $t \bar{t}$ production cross section. Diboson inclusive cross sections has been evaluated at NLO [66]. The $W^{ \pm} Z$ cross section increases from $23 \mathrm{pb}$ at LHC8 to $47 \mathrm{pb}$ at the LHC13, i.e., roughly by a factor of two 10

The naive estimate of the sensitivity reach of the LHC13 in this channel is obtained by scaling the expected exclusion sensitivity at $8 \mathrm{TeV}$ c.m.e., ${ }^{\mathrm{LHC} 8} \sigma_{\text {excl }}$, by a factor

$$
R_{13 / 8}=\sqrt{R_{\mathrm{bkg}}} \times \frac{\mathcal{L}_{\mathrm{LHC} 8}}{\mathcal{L}_{\mathrm{LHC} 13}},
$$

where the first term on the right hand side takes into account the increase of the background, which decreases the sensitivity, while the second term the increase of the signal with the luminosity. In our specific case this results in a factor of $\sqrt{2} \times \sqrt{21 / 100} \approx 0.65$, i.e. an improvement of $35 \%$ w.r.t. the current sensitivity.

In Fig. 9 we show the LHC13 expectations corresponding to the results shown in Fig. 3 , To guide the eye the dashed black line shows the LHC8 production cross section, and the dotted red line corresponds to the current exclusion, (i.e. the solid red in Fig. 3), whereas the solid red line in the four plots of Fig. 9 show the LHC13 expected exclusion obtained as described above. One can observe that the analysis neglecting $\tilde{\chi}_{2}^{0} \rightarrow \tilde{\chi}_{1}^{0} h_{1}$ would exclude all $m_{\tilde{\chi}_{2}^{0}}$ values in the parameter space analyzed. Taking the decay to $\tilde{\chi}_{1}^{0} h_{1}$ into account, however, the bound lies roughly between $m_{\tilde{\chi}_{2}^{0}} \sim 200 \mathrm{GeV}$ and $\sim 300 \mathrm{GeV}$, depending on $m_{\tilde{\chi}_{1}^{0}}$ and the sign of $M_{1}$.

The LHC13 expectations are similar for $\tan \beta=20$ as shown in Fig. 10, In agreement with Fig. 4 the excluded regions are larger than for $\tan \beta=6$, and for $m_{\tilde{\chi}_{1}^{0}} \geq 50 \mathrm{GeV}$ and $M_{1}$ negative no bound on $m_{\tilde{\chi}_{2}^{0}}$ can be read of (without extrapolation).

As for the LHC8 analysis we summarize the results of Figs. 9, 10 in contour plots in the $m_{\tilde{\chi}_{2}^{0}}-m_{\tilde{\chi}_{1}^{0}}$ plane shown in Fig. 11 for $\tan \beta=6$ (upper plot) and $\tan \beta=20$ (lower plot).

\footnotetext{
${ }^{10}$ The extrapolation of production cross sections to higher energies of both the background as well as the signal will depend on the specific choice of kinematical cuts. For instance, the ratio $R_{\text {bkg }}$ increases by up to $20 \%$ if one applies large transverse momentum cuts 66 . However, also the signal is expected to grow by a similar factor. Therefore we neglect these effects here.
} 

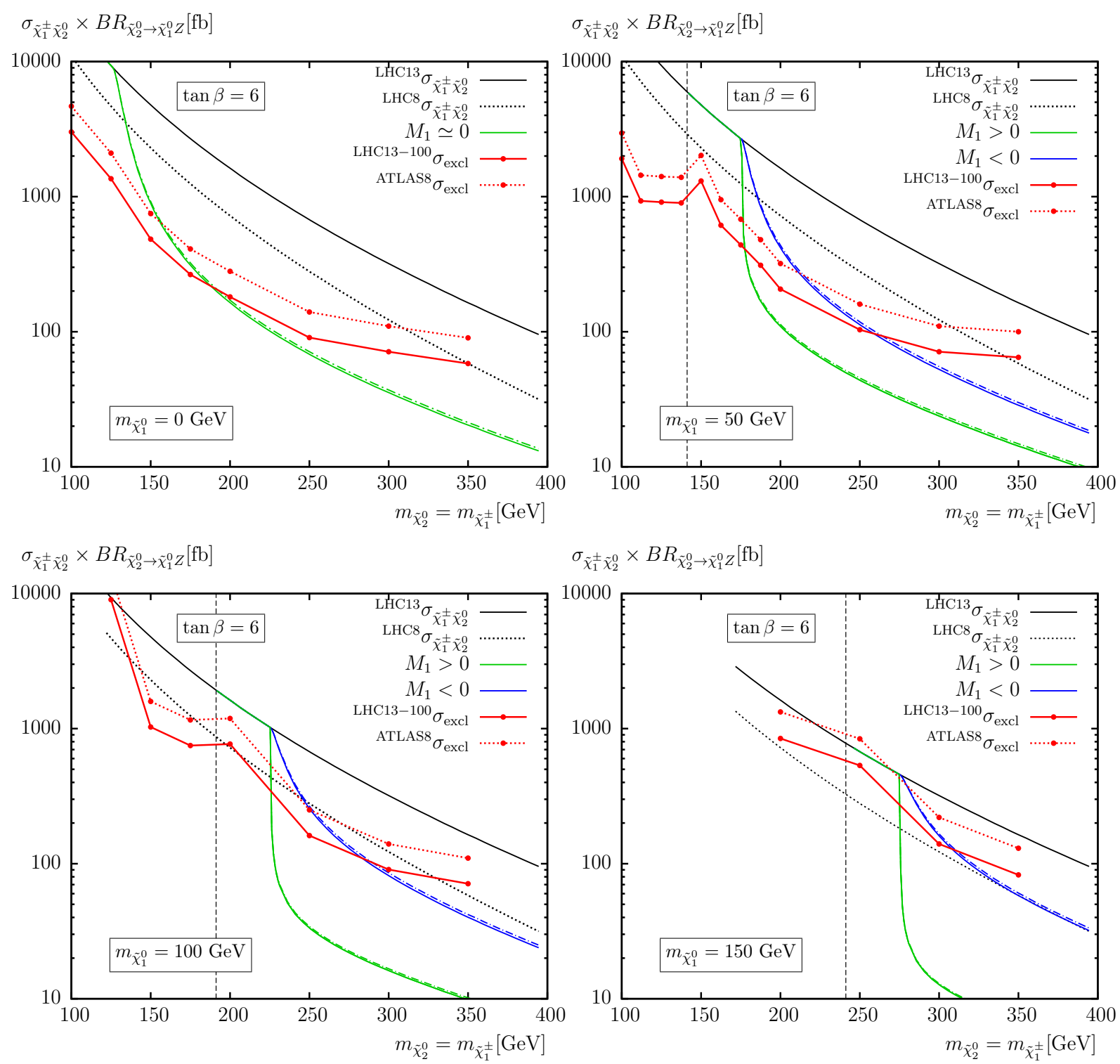

Figure 9: $\quad \tilde{\chi}_{1}^{ \pm}, \tilde{\chi}_{2}^{0}$ production cross section at the LHC13 evaluated with Prospino 2.1 [23] (black) times the $\operatorname{BR}\left(\tilde{\chi}_{2}^{0} \rightarrow Z \tilde{\chi}_{1}^{0}\right)$ at tree (solid) and one-loop level (dashed-dotted) for $M_{1}>0$ (green) and $M_{1}<0$ (blue). Also shown is the LHC8 production cross section (thin black). $M_{1}$ is chosen such that $m_{\tilde{\chi}_{1}^{0}}=0,50,100,150 \mathrm{GeV}$, top left and right, bottom left and right, respectively. The $95 \% \mathrm{CL}$ exclusion cross sections from ATLAS at $8 \mathrm{TeV}$ and $21 \mathrm{fb}^{-1}$ (solid red), 20] and the projection for 100 and $300 \mathrm{fb}^{-1}$ at LHC13 (dashed and dot-dashed red).

Here we compare the most recent ATLAS results with the LHC13 projections. The color coding is as in Fig. 5. In the low $\tan \beta$ case, even at the LHC13 only relatively small strips going beyond the region where $\tilde{\chi}_{2}^{0} \rightarrow \tilde{\chi}_{1}^{0} h_{1}$ is kinematically forbidden can be excluded. In the high $\tan \beta$ case the excluded values of $m_{\tilde{\chi}_{2}^{0}}$ for $m_{\tilde{\chi}_{1}^{0}} \sim 0$ for LHC13, taking $\tilde{\chi}_{2}^{0} \rightarrow \tilde{\chi}_{1}^{0} h_{1}$ into account, are similar to the limits currently published in Ref. [20], i.e. only with the LHC13 

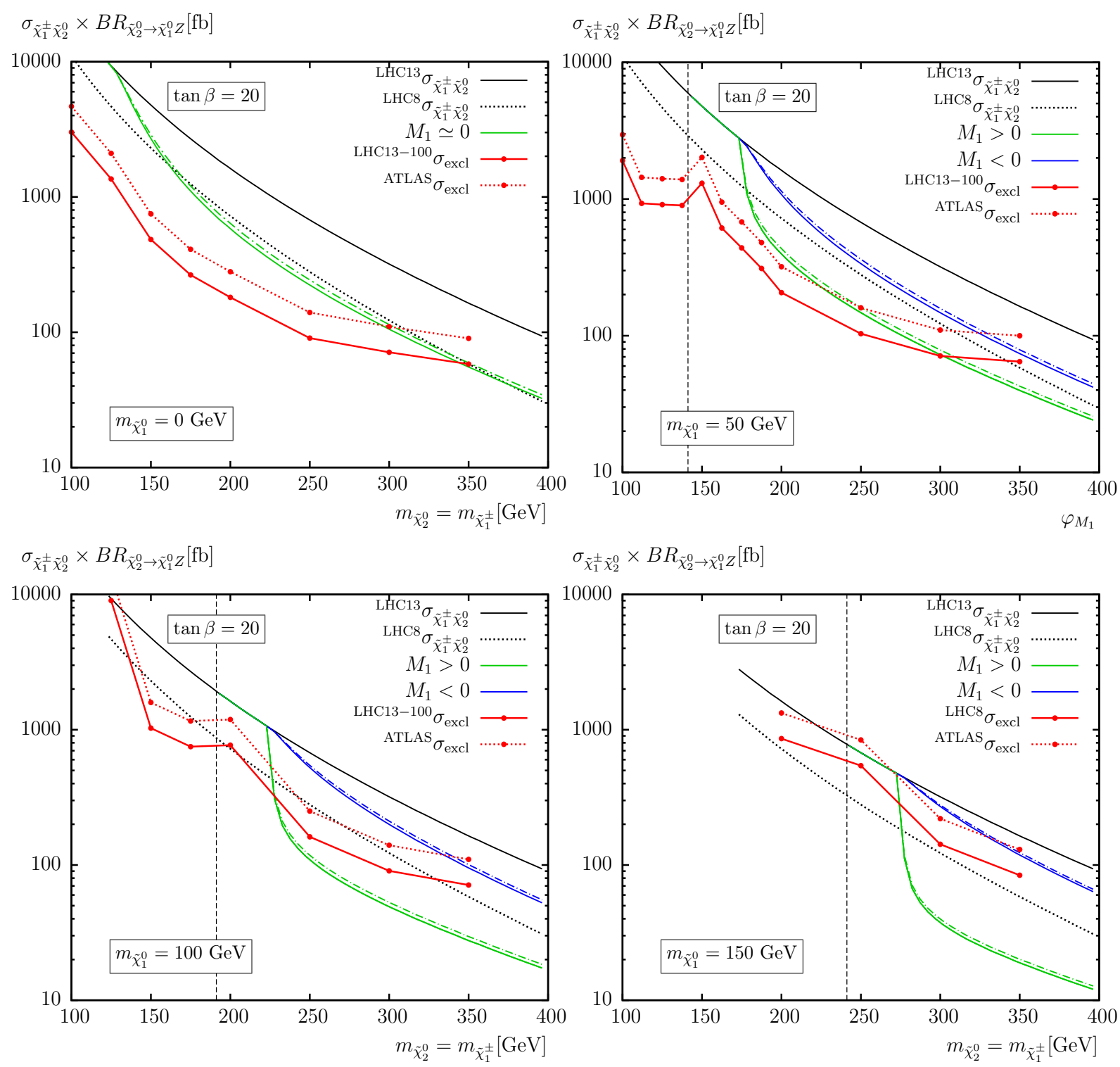

Figure 10: As in Fig. 9, but for $\tan \beta=20$.

the current simplified models exclusion can be reached. The same holds for positive $M_{1}$ for larger values of $m_{\tilde{\chi}_{1}^{0}}$.

The sensitivity of chargino/neutralino searches at the LHC13 has been discussed in the existing literature and can be related to our estimates. Searches for the decay $\tilde{\chi}_{2}^{0} \rightarrow \tilde{\chi}_{1}^{0} h_{1}$ will start to become sensitive [15,17, 60, 64]. This will provide an interesting complementary channel, which may well dominate completely over $\tilde{\chi}_{2}^{0} \rightarrow \tilde{\chi}_{1}^{0} Z$. It has been shown in Refs. [15, 17] that with $100 \mathrm{fb}^{-1}$, a $5 \sigma$ discovery should be possible for $m_{\tilde{\chi}_{2}^{0}} \sim 400 \ldots 500 \mathrm{GeV}$ (where the GUT relation between $M_{1}$ and $M_{2}$ has been assumed). This might overcome the reduction in sensitivity that we found in our analysis. It should finally be noticed that the branching ratio $\operatorname{BR}\left(\tilde{\chi}_{2}^{0} \rightarrow \tilde{\chi}_{1}^{0} h_{1}\right)$ may also be strongly dependent on $\mathcal{C P}$ phases and may in this way provide unique information on the neutralino and Higgs sectors. 

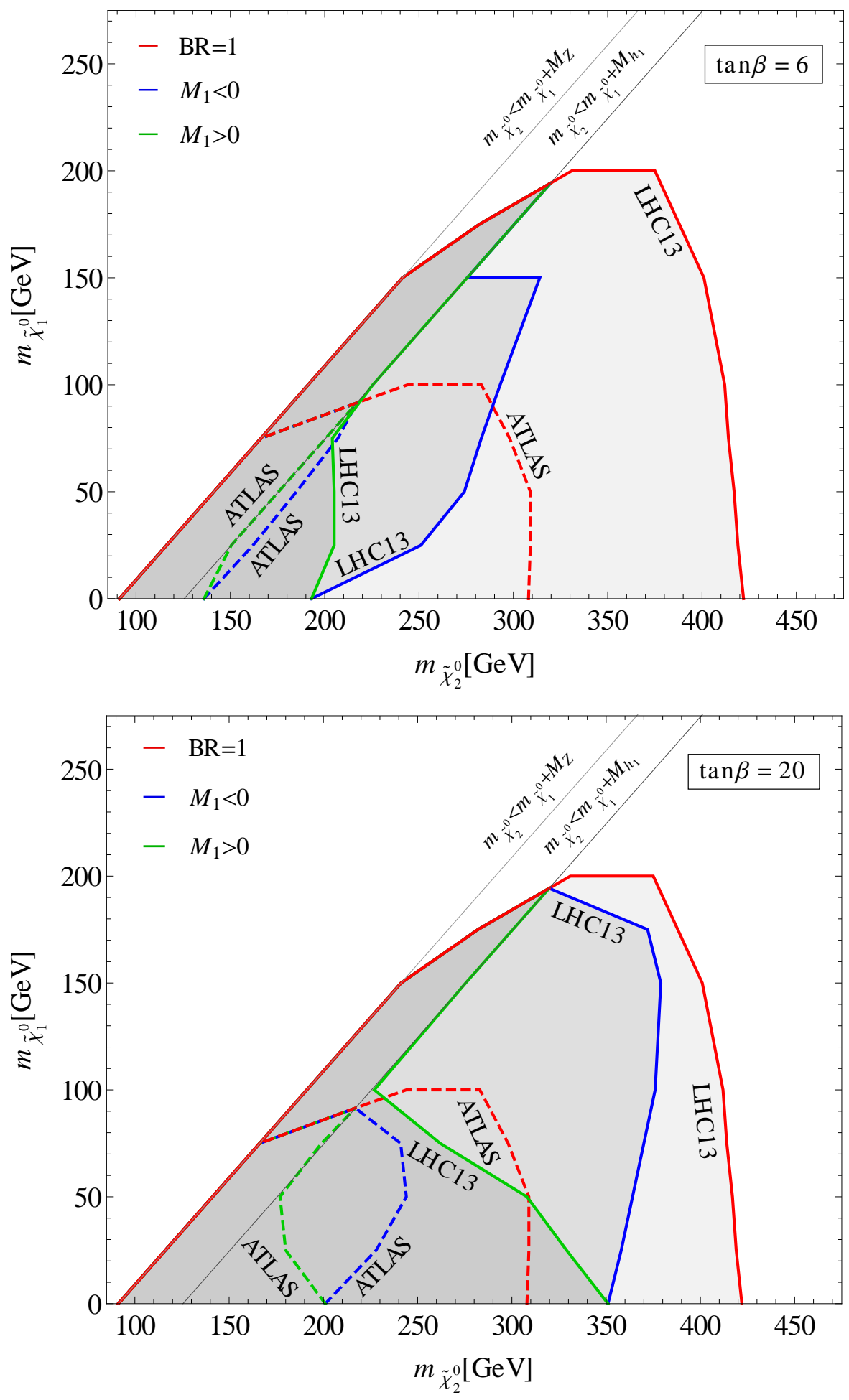

Figure 11: Contour plots showing approximate excluded region from $S_{\text {ATLAS }}$ in the $m_{\tilde{\chi}_{2}^{0-}}$ $m_{\tilde{\chi}_{1}^{0}}$ plane, with $\tan \beta=6$ (upper plot) and $\tan \beta=20$ (lower plot), showing the projected exclusions for $100 \mathrm{fb}^{-1}$ at LHC13. The color coding is as in Fig. [5. 


\subsection{Three-body decays of $\tilde{\chi}_{2}^{0}$}

In the region where the third lepton (the lepton not part of the SFOS lepton pair) is softest, ATLAS is supposedly sensitive to the decays via off-shell gauge bosons, and limits have been set, allowing the region close to the diagonal in the plane of the masses of the lightest neutralino and chargino to be probed. 11 The relevant decay channels are

$$
\tilde{\chi}_{2}^{0} \rightarrow \tilde{\chi}_{1}^{0} Z^{*} \rightarrow \tilde{\chi}_{1}^{0} l^{+} l^{-} \text {and } \tilde{\chi}_{2}^{0} \rightarrow \tilde{\ell}^{ \pm *} l^{\mp} \rightarrow \tilde{\chi}_{1}^{0} l^{+} l^{-}
$$

where it is crucial that the experimental searches are optimized for the $\tilde{\chi}_{2}^{0} \rightarrow \tilde{\chi}_{1}^{0} Z^{*}$ channel.

In this section we discuss some problems in the re-interpretation of the ATLAS limits from [20]. We would mainly like to point out that in the region below the $Z$ threshold, while it is a reasonable approximation to neglect the contribution via off-shell decays to Higgs bosons, there is substantial destructive interference between the off-shell $Z$ and slepton channels for slepton masses below approximately $5|\mu|$ (here $5 \mathrm{TeV}$ ). This was previously discussed in Ref. 67] and references therein. Within the ATLAS analysis the slepton channel was neglected, effectively by pushing the slepton masses to the multi-10 TeV scale. However, within most realizations of low-energy SUSY such high slepton mass scales are considered unrealistic, and masses not far above the LEP limits of $\mathcal{O}(100 \mathrm{GeV})$ are experimentally permitted - i.e. the interpretation of these bounds in terms of concrete models is difficult.

To illustrate this point, we have calculated the ratio of the partial decay width of $\tilde{\chi}_{2}^{0} \rightarrow$ $\tilde{\chi}_{1}^{0} l^{+} l^{-}$either excluding those diagrams involving sleptons or excluding diagrams involving $Z / h_{1}$ bosons, to the partial width including all possible diagrams,

$$
R_{Z}=\frac{\Gamma^{\mathrm{no}} \tilde{l}\left(\tilde{\chi}_{2}^{0} \rightarrow \tilde{\chi}_{1}^{0} l^{+} l^{-}\right)}{\Gamma^{\operatorname{total}}\left(\tilde{\chi}_{2}^{0} \rightarrow \tilde{\chi}_{1}^{0} l^{+} l^{-}\right)} \quad \text { and } \quad R_{\tilde{l}}=\frac{\Gamma^{\text {no } Z h_{1}}\left(\tilde{\chi}_{2}^{0} \rightarrow \tilde{\chi}_{1}^{0} l^{+} l^{-}\right)}{\Gamma^{\operatorname{total}}\left(\tilde{\chi}_{2}^{0} \rightarrow \tilde{\chi}_{1}^{0} l^{+} l^{-}\right)}
$$

for $l=e$ and $\mu$. The results are shown for the ATLAS baseline scenarios in Fig. 12 for $\tan \beta=6$ ( $S_{\text {ATLAS }}$, left) and $\tan \beta=20$ ( $S_{\text {ATLAS }}^{\tan \beta}$, right). $R_{Z}\left(R_{\tilde{\ell}}\right)$ is shown as solid (dashed) lines for $M_{1}>0$ (blue) and $M_{1}<0$ (green). Note that this interference is sensitive to $\mu$, and the position of the peak of this quantity is close to the value of $\mu$, which in $S_{\text {ATLAS }}$ is $1 \mathrm{TeV}$. Note furthermore that the coupling $\tilde{\chi}_{2}^{0} \rightarrow \tilde{\chi}_{1}^{0} Z$ is inversely proportional to $\mu^{2}$, see Eq. (77). On the other hand the slepton propagator renders a factor proportional to $1 / M_{\tilde{\ell}}^{2}$ in the amplitude for the decay via sleptons. Consequently, in the region where $\mu \sim M_{\tilde{\ell}}$, the interference is maximal, as can be observed in Fig. 12. Further, as the slepton mass increases, the contribution of the sleptons decouples, and $R_{Z}$ approaches 1 , whereas $R_{\tilde{\ell}}$ approaches zero, as expected. A similar interference effect might also occur below the threshold for the chargino decay $\tilde{\chi}_{1}^{ \pm} \rightarrow \tilde{\chi}_{1}^{0} W^{-}$, however a detailed study is beyond the scope of this paper. In summary, Fig. 12 shows that the slepton mass scale has a dramatic effect on the composition of the decay channel $\tilde{\chi}_{2}^{0} \rightarrow \tilde{\chi}_{1}^{0} l^{+} l^{-}$, which is crucial for the experimental analysis. Consequently, the excluded regions should be viewed with care and not taken at face value. The published limits are only valid for (normally deemed unrealistic) relatively high slepton mass scales.

\footnotetext{
${ }^{11}$ This area was not marked in our contour plots in Fig. 5, 11.
} 

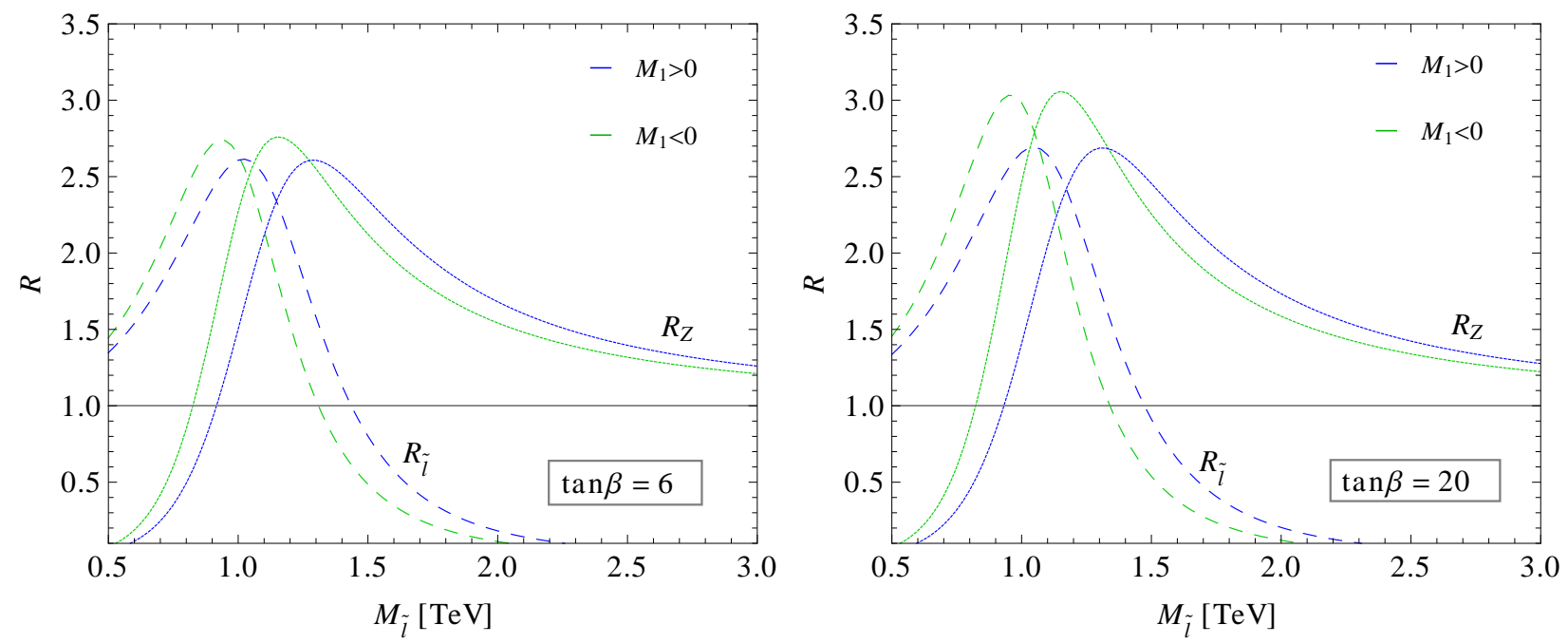

Figure 12: We show the ratios $R_{Z}$ (solid) and $R_{\tilde{l}}$ (dashed), as defined in Eq. (20) as a function of $M_{\tilde{\ell}}$ for $\tan \beta=6$ (left) and $\tan \beta=20$ (right). Results for $M_{1}>0$ are shown in blue, for $M_{1}<0$ in green. All other parameters are as for our ATLAS baseline scenario.

\section{Conclusions}

We re-assessed the exclusion limits on the parameters describing the supersymmetric (SUSY) electroweak sector of the MSSM obtained from the search for direct $\tilde{\chi}_{1}^{ \pm} \tilde{\chi}_{2}^{0}$ production via $W Z+E_{T}^{\text {miss }}$ at the LHC. Our starting point is the baseline scenario used by ATLAS in their most recent public note using $21 \mathrm{fb}^{-1}[20$. This analysis is carried out for a simplified model scenario where it is assumed that the $\tilde{\chi}_{1}^{ \pm}$and $\tilde{\chi}_{2}^{0}$ decay $100 \%$ via $\tilde{\chi}_{1}^{ \pm} \rightarrow \tilde{\chi}_{1}^{0} W^{ \pm}$and $\tilde{\chi}_{2}^{0} \rightarrow \tilde{\chi}_{1}^{0} Z$, and limits on $m_{\tilde{\chi}_{2}^{0}}$ of up to $\sim 300 \mathrm{GeV}$ are derived, mostly displayed in the $m_{\tilde{\chi}_{2}^{0}}-m_{\tilde{\chi}_{1}^{0}}$ plane. In our analysis we investigated how these limits change on using NLO results both for the SUSY production cross sections as well as for the branching ratio calculations.

The first step in our analysis was the inclusion of the decay $\tilde{\chi}_{2}^{0} \rightarrow \tilde{\chi}_{1}^{0} h_{1}$, which can substantially lower $\operatorname{BR}\left(\tilde{\chi}_{2}^{0} \rightarrow \tilde{\chi}_{1}^{0} Z\right)$, thereby strongly reducing the excluded parameter space. Besides the region where $\tilde{\chi}_{2}^{0} \rightarrow \tilde{\chi}_{1}^{0} h_{1}$ is kinematically forbidden, only a very small strip in the $m_{\tilde{\chi}_{2}^{0}}-m_{\tilde{\chi}_{1}^{0}}$ plane can be excluded. As a second step we allowed the gaugino mass parameter $M_{1}$ to take negative values (corresponding to $\varphi_{M_{1}}=\pi$ ). In this case slightly larger regions in the $m_{\tilde{\chi}_{2}^{0}}-m_{\tilde{\chi}_{1}^{0}}$ plane can be excluded. Going from the baseline value $\tan \beta=6$ to $\tan \beta=20$ again leads to somewhat larger excluded regions, but the decay $\tilde{\chi}_{2}^{0} \rightarrow \tilde{\chi}_{1}^{0} h_{1}$ is still clearly seen to have a substantial effect on the limits.

We additionally assessed how much a combination of ATLAS and CMS data could change the results (effectively assuming a doubling of the analyzed ATLAS data). Only in the most "favorable" case, $\tan \beta=20$ and $\varphi_{M_{1}}=\pi$ the combined ATLAS/CMS analysis nearly reaches the exclusion region reported by ATLAS alone for the simplified model case.

As the next step we investigated the dependence of the excluded mass regions on the phase of $M_{1}$. By projecting the results onto the $\varphi_{M_{1}}-M_{1}$ plane a strong dependence on $\varphi_{M_{1}}$ becomes evident. In the future, limits on $W Z+E_{T}^{\mathrm{miss}}$ and $W h+E_{T}^{\mathrm{miss}}$ could also be exploited 
as a method to constrain $\varphi_{M_{1}}$, complementary to the EDMs. Furthermore, the relevance of the loop corrections in the branching ratio calculations was shown to reach more than $10 \mathrm{GeV}$ in the limit on $M_{1}$, amounting up to a change of $30 \%$. The overall size of the NLO corrections in the branching ratio calculations was found to reach the level of up to $12 \%$.

Another interesting deviation from the ATLAS baseline scenario is given by the inclusion of a light scalar tau, with $m_{\tilde{\tau}_{1}}-m_{\tilde{\chi}_{1}^{0}} \lesssim 10 \mathrm{GeV}$, such that the $\tilde{\chi}_{1}^{0}$ provides the correct amount of relic Cold Dark Matter [3, $\left[57\right.$. This opens up the decay modes $\tilde{\chi}_{2}^{0} \rightarrow \tilde{\tau}_{1} \tau$ and $\tilde{\chi}_{1}^{ \pm} \rightarrow \tilde{\tau}_{1}^{ \pm} \nu_{\tau}$, further reducing the desired branching ratios for $\tilde{\chi}_{2}^{0} \rightarrow \tilde{\chi}_{1}^{0} Z$. We have shown that the new decay modes can strongly influence the parameter dependences of $\operatorname{BR}\left(\tilde{\chi}_{2}^{0} \rightarrow \tilde{\chi}_{1}^{0} Z\right)$ and thus require a new analysis in this scenario where the searches for different relevant decays modes are combined.

The final scenario analyzed has $M_{1} \leq \mu \leq M_{2}$, leading to higgsino-like neutralinos $\tilde{\chi}_{2}^{0}$, $\tilde{\chi}_{3}^{0}$, and a higgsino-like chargino $\tilde{\chi}_{1}^{ \pm}$with $m_{\tilde{\chi}_{2}^{0}} \approx m_{\tilde{\chi}_{3}^{0}} \approx m_{\tilde{\chi}_{1}^{ \pm}}$. In this scenario the lower value of $\mu$ results in strongly reduced production cross sections, and no mass value can be excluded by re-analyzing the published bounds. In this scenario a combination not only of ATLAS and CMS data, but also of the production modes $\tilde{\chi}_{1}^{ \pm} \tilde{\chi}_{2}^{0}$ and $\tilde{\chi}_{1}^{ \pm} \tilde{\chi}_{3}^{0}$ will have to be performed to reach sensitivity for higgsino masses below $\sim 200 \mathrm{GeV}$.

As a last step we presented the exclusion regions expected for $100 \mathrm{fb}^{-1}$ analyzed by ATLAS during the next LHC run at $\sqrt{s}=13 \mathrm{TeV}$, assuming the absence of any signal. We showed that with the strong increase in the integrated luminosity as well as with the increase in the production cross sections, the current simplified model exclusion regions can roughly be reached, where details depend on the $\varphi_{M_{1}}$ and $\tan \beta$. This would result in an important advance into MSSM parameter space, and any hints of low charginos-neutralinos seen could further be investigated at the linear collider [35.

Finally, we briefly investigated the regions where ATLAS claims sensitivity to $\tilde{\chi}_{1}^{ \pm} \tilde{\chi}_{2}^{0}$ production via the off-shell decay $\tilde{\chi}_{2}^{0} \rightarrow \tilde{\chi}_{1}^{0} l^{+} l^{-}$, i.e. below the kinematic threshold for $\tilde{\chi}_{2}^{0} \rightarrow$ $\tilde{\chi}_{1}^{0} Z$. We showed that more realistic values for the slepton mass scale can strongly enhance the interference between $\tilde{\chi}_{2}^{0} \rightarrow \tilde{\chi}_{1}^{0} Z^{*} \rightarrow \tilde{\chi}_{1}^{0} l^{+} l^{-}$and $\tilde{\chi}_{2}^{0} \rightarrow \tilde{\ell}^{ \pm} l^{ \pm} \rightarrow \tilde{\chi}_{1}^{0} l^{+} l^{-}$. This in turn will have a strong impact on the lepton distributions and thus on the experimental analysis in this kinematic region.

In summary we have re-analyzed the latest ATLAS limits on direct electroweak SUSY production. We have found that translating the public limits obtained in simplified scenarios to realistic scenarios, where in particular the decay $\tilde{\chi}_{2}^{0} \rightarrow \tilde{\chi}_{1}^{0} h_{1}$ is accounted for, strongly reduced the excluded parameter regions. Conversely, we encourage the LHC experiments to include the channel $\tilde{\chi}_{2}^{0} \rightarrow \tilde{\chi}_{1}^{0} h_{1}$ into their analysis, as this would provide access to SUSYHiggs couplings and strengthen the electroweak SUSY searches.

\section{Acknowledgments}

We thank A. Calderón, J. Dietrich, M. Elsing, A. Höcker, N. Kauer, F. Moortgat, G. MoortgatPick, T. Potter, W. Waltenberger and G. Weiglein for helpful discussions. A.B. gratefully acknowledges support of the DFG through the grant SFB 676, "Particles, Strings, and the Early Universe". The work of S.H. was partially supported by CICYT (grant FPA 201022163-C02-01). F.v.d.P. was supported by the Spanish MICINN's Consolider-Ingenio 2010 
Programme under grant MultiDark CSD2009-00064. We thank the GRID computing network at IFCA for technical help with the OpenStack cloud infrastructure.

\section{References}

[1] H. Nilles, Phys. Rept. 110 (1984) 1;

H. Haber and G. Kane, Phys. Rept. 117 (1985) 75;

R. Barbieri, Riv. Nuovo Cim. 11 (1988) 1.

[2] H. Dreiner, In *Kane, G.L. (ed.): Perspectives on supersymmetry II* 565-583 arXiv:hep-ph/9707435].

[3] H. Goldberg, Phys. Rev. Lett. 50 (1983) 1419;

J. Ellis, J. Hagelin, D. Nanopoulos, K. Olive and M. Srednicki, Nucl. Phys. B 238 (1984) 453.

[4] G. Aad et al. [The ATLAS Collaboration], arXiv:1207.7214 [hep-ex];

S. Chatrchyan et al. [The CMS Collaboration], arXiv:1207.7235 [hep-ex].

[5] R. Mahbubani, M. Papucci, G. Perez, J. Ruderman and A. Weiler, arXiv:1212.3328 [hep-ph].

[6] S. AbdusSalam, B. Allanach, H. Dreiner, J. Ellis, U. Ellwanger, J. Gunion, S. Heinemeyer and M. Krämer et al., Eur. Phys. J. C 71 (2011) 1835 [arXiv:1109.3859 [hep-ph]].

[7] M. Benayoun, P. David, L. DelBuono and F. Jegerlehner, arXiv:1210.7184 [hep-ph].

[8] G. Aad et al. [ATLAS Collaboration], Phys. Lett. B 718 (2013) 841 arXiv:1208.3144 [hep-ex]].

[9] S. Chatrchyan et al. [CMS Collaboration], arXiv:1209.6620 [hep-ex].

[10] H. Baer, C. -h. Chen, F. Paige and X. Tata, Phys. Rev. D 50 (1994) 4508 arXiv:hep-ph/9404212].

[11] H. Baer, V. Barger, S. Kraml, A. Lessa, W. Sreethawong and X. Tata, JHEP 1203 (2012) 092 arXiv:1201.5382 [hep-ph]].

[12] H. Baer, V. Barger and P. Huang, JHEP 1111 (2011) 031 [arXiv:1107.5581 [hep-ph]].

[13] H. Baer, V. Barger, P. Huang, D. Mickelson, A. Mustafayev, W. Sreethawong and X. Tata, arXiv:1302.5816 [hep-ph].

[14] M. Cabrera, J. Casas and B. Zaldivar, arXiv:1212.5247 [hep-ph].

[15] H. Baer, V. Barger, A. Lessa, W. Sreethawong and X. Tata, Phys. Rev. D 85 (2012) 055022 [arXiv:1201.2949 [hep-ph]].

[16] P. Byakti and D. Ghosh, Phys. Rev. D 86 (2012) 095027 [arXiv:1204.0415 [hep-ph]]. 
[17] D. Ghosh, M. Guchait and D. Sengupta, Eur. Phys. J. C 72 (2012) 2141 arXiv:1202.4937 [hep-ph]].

[18] [ATLAS Collaboration], ATLAS-CONF-2012-154.

[19] [ATLAS Collaboration], ATLAS-CONF-2013-028.

[20] [ATLAS Collaboration], ATLAS-CONF-2013-035.

[21] [ATLAS Collaboration], ATLAS-CONF-2013-049.

[22] S. Chatrchyan et al. [CMS Collaboration], CMS PAS SUS-12-022.

[23] W. Beenakker, M. Klasen, M. Kramer, T. Plehn, M. Spira and P. Zerwas, Phys. Rev. Lett. 83 (1999) 3780 [Erratum-ibid. 100 (2008) 029901] [arXiv:hep-ph/9906298].

[24] B. Fuks, M. Klasen, D. R. Lamprea and M. Rothering, JHEP 1210 (2012) 081 arXiv:1207.2159 [hep-ph]].

[25] B. Fuks, M. Klasen, D. R. Lamprea and M. Rothering, Eur. Phys. J. C 73 (2013) 2480 arXiv:1304.0790 [hep-ph]].

[26] N. Baro and F. Boudjema, Phys. Rev. D 80 (2009) 076010 [arXiv:0906.1665 [hep-ph]].

[27] J. Fujimoto, T. Ishikawa, Y. Kurihara, M. Jimbo, T. Kon and M. Kuroda, Phys. Rev. D 75 (2007) 113002.

[28] S. Liebler and W. Porod, Nucl. Phys. B 849 (2011) 213 [Erratum-ibid. B 856 (2012) 125] arXiv:1011.6163 [hep-ph]].

[29] M. Drees, W. Hollik and Q. Xu, JHEP 0702 (2007) 032 [arXiv:hep-ph/0610267].

[30] W. Yang and D. Du, Phys. Rev. D 67 (2003) 055004 arXiv:hep-ph/0211453.

[31] H. Eberl, T. Gajdosik, W. Majerotto and B. Schrausser, Phys. Lett. B 618 (2005) 171 arXiv:hep-ph/0502112].

[32] A. Bharucha, A. Fowler, G. Moortgat-Pick and G. Weiglein, JHEP 1305 (2013) 053 arXiv:1211.3134 [hep-ph]].

[33] S. Heinemeyer, F. von der Pahlen and C. Schappacher, Eur. Phys. J. C 72 (2012) 1892 arXiv:1112.0760 [hep-ph]].

[34] A. Bharucha, S. Heinemeyer, F. von der Pahlen and C. Schappacher, Phys. Rev. D 86 (2012) 075023 arXiv:1208.4106 [hep-ph]].

[35] A. Bharucha, J. Kalinowski, G. Moortgat-Pick, K. Rolbiecki and G. Weiglein, arXiv:1211.3745 [hep-ph].

[36] T. Takagi, Japan J. Math. 1 (1925) 83. 
[37] V. Barger, T. Falk, T. Han, J. Jiang, T. Li and T. Plehn, Phys. Rev. D 64 (2001) 056007 arXiv:hep-ph/0101106.

[38] A. Pilaftsis, Phys. Rev. D 58 (1998) 096010 arXiv:hep-ph/9803297];

A. Pilaftsis, Phys. Lett. B 435 (1998) 88 [arXiv:hep-ph/9805373].

[39] D. Demir, Phys.Rev. D 60 (1999) 055006 [arXiv:hep-ph/9901389].

[40] A. Pilaftsis and C. Wagner, Nucl. Phys. B 553 (1999) 3 arXiv:hep-ph/9902371.

[41] S. Heinemeyer, Eur. Phys. J. C 22 (2001) 521 arXiv:hep-ph/0108059.

[42] S. Heinemeyer, W. Hollik and G. Weiglein, Comput. Phys. Commun. 124 (2000) 76 arXiv:hep-ph/9812320];

T. Hahn, S. Heinemeyer, W. Hollik, H. Rzehak and G. Weiglein, Comput. Phys. Commun. 180 (2009) 1426; see www.feynhiggs.de.

[43] S. Heinemeyer, W. Hollik and G. Weiglein, Eur. Phys. J. C 9 (1999) 343 arXiv:hep-ph/9812472].

[44] G. Degrassi, S. Heinemeyer, W. Hollik, P. Slavich and G. Weiglein, Eur. Phys. J. C 28 (2003) 133 arXiv:hep-ph/0212020.

[45] M. Frank, T. Hahn, S. Heinemeyer, W. Hollik, R. Rzehak and G. Weiglein, JHEP 0702 (2007) 047 arXiv:hep-ph/0611326.

[46] The couplings can be found in the files MSSM.ps.gz, MSSMQCD.ps.gz and HMix.ps.gz as part of the FeynArts package [48].

[47] A. Dobado, M. Herrero and S. Peñaranda, Eur. Phys. J. C 17 (2000) 487 arXiv:hep-ph/0002134;

J. Gunion and H. Haber, Phys. Rev. D 67 (1993) 075019 arXiv:hep-ph/0207010;

H. Haber and Y. Nir, Phys. Lett. B 306 (1993) 327 [arXiv:hep-ph/9302228];

H. Haber, arXiv:hep-ph/9505240.

[48] J. Küblbeck, M. Böhm and A. Denner, Comput. Phys. Commun. 60 (1990) 165;

T. Hahn, Comput. Phys. Commun. 140 (2001) 418 arXiv:hep-ph/0012260];

T. Hahn and C. Schappacher, Comput. Phys. Commun. 143 (2002) 54 arXiv:hep-ph/0105349].

The program, the user's guide and the MSSM model files are available via www. feynarts.de .

[49] T. Hahn and M. Pérez-Victoria, Comput. Phys. Commun. 118 (1999) 153 arXiv:hep-ph/9807565.

[50] I. Campos, E. del Castillo, S. Heinemeyer, A. Lopez-Garcia and F. von der Pahlen, Eur. Phys. J. C 73 (2013) 2375 arXiv:1212.4784 [cs.DC]].

[51] C. Baker et al., Phys. Rev. Lett. 97 (2006) 131801 arXiv:hep-ex/0602020]. 
[52] B. Regan, E. Commins, C. Schmidt and D. DeMille, Phys. Rev. Lett. 88 (2002) 071805.

[53] W. Griffith, M. Swallows, T. Loftus, M. Romalis, B. Heckel and E. Fortson, Phys. Rev. Lett. 102 (2009) 101601.

[54] J. Lee, M. Carena, J. Ellis, A. Pilaftsis and C. Wagner, Comput. Phys. Commun. 184 (2013) 1220 arXiv:1208.2212 [hep-ph]].

[55] J. Lee, M. Carena, J. Ellis, A. Pilaftsis and C. Wagner, Comput. Phys. Commun. 180 (2009) 312 [arXiv:0712.2360 [hep-ph]].

[56] J. Lee, A. Pilaftsis, M. Carena, S. Choi, M. Drees, J. Ellis and C. Wagner, Comput. Phys. Commun. 156 (2004) 283 [arXiv:hep-ph/0307377.

[57] G. Belanger, F. Boudjema, A. Pukhov and A. Semenov, arXiv:1305.0237 [hep-ph]; arXiv:1005.4133 [hep-ph]; arXiv:0803.2360 [hep-ph]; Comput. Phys. Commun. 176 (2007) 367 [arXiv:hep-ph/0607059].

[58] P. Ade et al. [Planck Collaboration], arXiv:1303.5076 [astro-ph.CO].

[59] H. Dreiner, S. Heinemeyer, O. Kittel, U. Langenfeld, A. Weber and G. Weiglein, Eur. Phys. J. C 62 (2009) 547 [arXiv:0901.3485 [hep-ph]].

[60] K. Howe and P. Saraswat, JHEP 1210, 065 (2012) [arXiv:1208.1542 [hep-ph]].

[61] D. Demir, O. Lebedev, K. A. Olive, M. Pospelov and A. Ritz, Nucl. Phys. B 680 (2004) 339 [arXiv:hep-ph/0311314].

[62] J. Ellis, J. S. Lee and A. Pilaftsis, JHEP 1102, 045 (2011) [arXiv:1101.3529 [hep-ph]].

[63] M. Pospelov and A. Ritz, Annals Phys. 318 (2005) 119 [hep-ph/0504231].

[64] A. Arbey, M. Battaglia and F. Mahmoudi, arXiv:1212.6865 [hep-ph].

[65] J.Olsen, "CMS future plans", talk given at Snowmass: Seattle Energy Frontier Workshop, July 1, 2013, https://indico.fnal.gov/getFile. py/access? contribId=10\&sessionId=0\& resId=0\&material Id=slides\&conf $I d=6969$.

[66] J. Campbell, R. Ellis and C. Williams, JHEP 1107 (2011) 018 arXiv:1105.0020 [hep$\mathrm{ph}]]$.

[67] H. Baer and X. Tata, Phys. Rev. D 47 (1993) 2739. 